\title{
35. GEOCHEMISTRY OF PERIPLATFORM CARBONATE SEDIMENTS, LEG 115, SITE 716 (MALDIVES ARCHIPELAGO, INDIAN OCEAN) ${ }^{1}$
}

\author{
Mitchell J. Malone, ${ }^{2}$ Paul A. Baker, ${ }^{2}$ Stephen J. Burns,${ }^{3}$ and Peter K. Swart ${ }^{3}$
}

\begin{abstract}
Site 716 is a continuous sequence (upper Miocene to Holocene) of periplatform oozes and chalks from the Maldives Ridge, Indian Ocean. Mineralogical and geochemical studies of these carbonate sediments indicate that submarine burial diagenesis has played an important role in the induration of sediments at this site. Metastable carbonates, high-magnesium calcite (HMC) and aragonite, convert to low-magnesium calcite (LMC) rapidly, within 1.1 and $6.0 \mathrm{Ma}$, respectively. Strontium concentrations in carbonate decrease with depth as the result of the burial diagenesis of calcium carbonate, primarily aragonite, with excess strontium being expelled into pore waters. The formation of celestite at depth indicates that sufficient diagenesis of carbonate sediments has occurred to saturate or supersaturate pore waters with respect to this authigenic mineral. Sodium also decreases monotonically with depth as a result of the burial diagenesis of calcium carbonate.

Magnesium and carbon and oxygen isotopic curves are remarkably similar. Carbon isotopic compositions record inputs of ${ }^{13} \mathrm{C}$-enriched components from shallow carbonate banks. Magnesium concentrations vary widely, recording enhanced episodes of cementation by LMC with slightly elevated magnesium contents. Positive shifts in oxygen isotopic composition also record episodes of cementation during burial diagenesis. Intervals with increased accumulation rates of metastable components have undergone more rapid diagenesis than intervals with predominately pelagic deposition.
\end{abstract}

\section{INTRODUCTION}

Periplatform oozes are transitional carbonate deposits found between carbonate banks and the deep ocean (Schlager and James, 1978). Mineralogically, they are a mixture of bank-derived sediment, primarily high-Sr aragonite and high-magnesium calcite (HMC) $\left(>4 \% \mathrm{MgCO}_{3}\right)$, plus pelagic ooze composed of primarily low-magnesium calcite (LMC) coccoliths and foraminifers (Schlager and James, 1978). In addition, low-Sr aragonite pteropods can be an important component. The periplatform setting provides an ideal environment to study the potentially rapid diagenetic alteration of metastable carbonate minerals in marine carbonate sediments. As noted by previous investigators, the more intensely studied deep-sea monomineralic pelagic oozes are not representative of many ancient limestones (Schlager and James, 1978; Baker, 1981). The submarine diagenesis of periplatform sediments provides a modern example of how many Paleozoic shelf and slope limestones formed. These deposits probably formed by the accumulation and subsequent diagenetic alteration of aragonite and HMC in shallow subsiding basins subjected to little freshwater diagenesis. The evolution of LMC pelagic organisms in the Mesozoic heralded a decrease in the importance of shallow-water limestones. Consequently, the study of the diagenesis of periplatform sediments may provide geologists with insight into the lithification of ancient limestones.

Previous studies of periplatform oozes have been located primarily in the Bahamas (Kier and Pilkey, 1971; Droxler et al., 1983; Boardman and Neumann, 1985; Boardman et al., 1986; Burns and Neumann, 1987; Droxler et al., 1988). These studies have concentrated principally on sedimentological aspects of

\footnotetext{
${ }^{1}$ Duncan, R. A., Backman, J., Peterson, L. C., et al., 1990. Proc. ODP, Sci. Results, 115: College Station, TX (Ocean Drilling Program).

2 Department of Geology, Duke University, P. O. Box 6729, College Station, Durham, NC 27708 , U.S.A.

3 Rosenstiel School for Marine and Atmospheric Sciences, Department of Geology and Geophysics, University of Miami, 4600 Rickenbacker Causeway, Mi. ami, FL 33149, U.S.A.
}

these deposits. Diagenetic studies of the Bahamian sediments include those of Schlager and James (1978), Mullins et al. (1980, 1985a), Dix and Mullins (1988a, 1988b), and Swart and Guzikowski (1988) (the only previous study of periplatform pore-water chemistry). Site 716 of ODP Leg 115 is one of only a few such periplatform sites ever drilled (the others were on ODP Leg 101 in the Bahamas). It is also the best of those sites in terms of having a nearly constant sedimentation rate and continuous recovery. Thus, Site 716 provides an excellent opportunity to increase our understanding of the diagenetic alteration of these sediments.

Site 716 is located in the Indian Ocean at $4^{\circ} 56.0^{\prime} \mathrm{N}$ and $73^{\circ}$ $17.0^{\prime} \mathrm{E}$ in a water depth of $543 \mathrm{~m}$. The site is situated in a broad, shallow basin on the Maldives Ridge between the double atoll chain of the Maldives Archipelago (Fig. 1). The basin is filled with about $2.2 \mathrm{~km}$ of sediments and sedimentary rocks. The site is located in the center of the chain about $20-30 \mathrm{~km}$ from the present-day banks. Hole $716 \mathrm{~B}$ is a $264.4-\mathrm{m}$ hydraulic piston core with $100 \%$ recovery. The core contains a continuous, apparently uninterrupted late Miocene to Holocene sedimentary record (Backman, Duncan, et al., 1988). It consists of a homogeneous and monotonous sediment; olive, and pale olive to olive grey in color with no distinct turbidite layers. The color changes are gradational and bioturbation is seldom observed. Generally, the sediments are foraminifer-bearing nannofossil oozes, with pteropods and aragonite needles occurring abundantly in the upper part of the core. The pteropods become less abundant downcore and have essentially disappeared by approximately $54 \mathrm{~m}$ below seafloor (mbsf; $1.4 \mathrm{Ma}$ ).

Sedimentation rates based on nannofossil datum levels are 56 $\mathrm{m} / \mathrm{m}$.y. for the late Miocene and early Pliocene, $22-23 \mathrm{~m} / \mathrm{m}$.y. for the late Pliocene, and $38 \mathrm{~m} / \mathrm{m}$.y. for the Pleistocene (Backman, Duncan, et al., 1988). This study will attempt to resolve the timing and nature of cementation at Site 716 by determining the chemical composition and isotopic signature of sediments and chalks at this site.

\section{METHODS}

The core was sampled throughout its entire length at $50-\mathrm{cm}$ intervals. In addition, samples of prominent chalk and celestite 


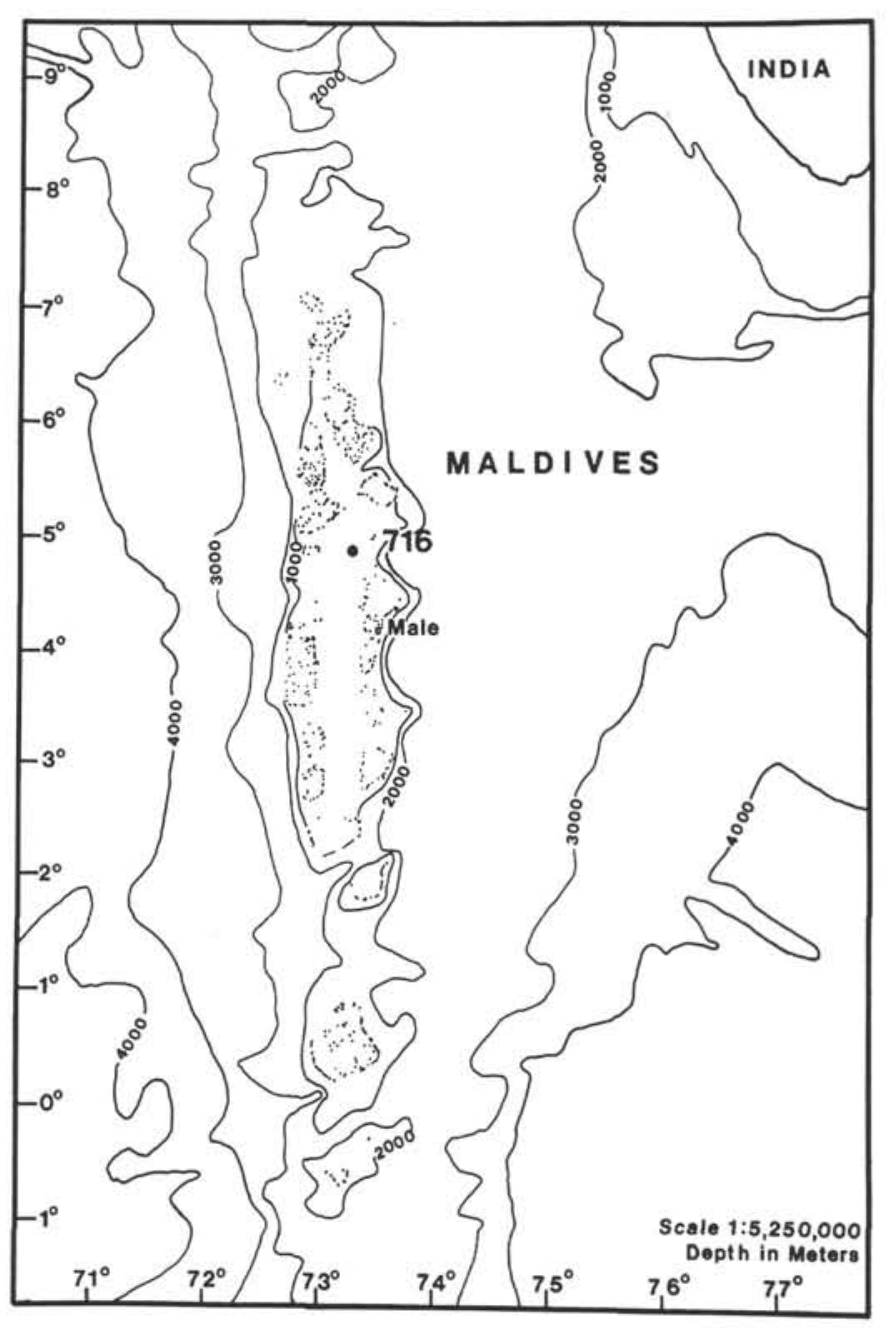

Figure 1. Location of Site 716.

nodules were taken. Chalks were identified by the methods of Gealy et al. (1971). According to their descriptive classification, chalks are firm or friable sediments that can be scratched with a fingernail or the edge of a spatula blade, whereas oozes have little strength and are easily deformed under the pressure of a finger or the broad blade of a spatula. This method was subjective and certainly some chalks could have been overlooked.

Approximately 550 samples were subjected to the following analyses. About $1 \mathrm{~g}$ of each sample was rinsed twice with distilled water, centrifuged, dried at $110^{\circ} \mathrm{C}$, and powdered. One fraction was used to determine mineralogy on 154 selected samples by powder X-ray diffraction using a Phillips X-ray diffractometer with $\mathrm{Cu}-\mathrm{K} \alpha$ radiation. Integrated peak area ratios of aragonite and calcite (low- $\mathrm{Mg}$ and high-Mg) were used to calculate their relative percentages vs. a standard calibration curve, as suggested by Milliman (1974). The LMC and HMC peaks were split following Milliman's technique (1974). The precision of these analyses was better than $5 \%$. A second fraction of each sample was leached in acetic acid buffered by ammonium-acetate $(\mathrm{pH}=5.5)$ for approximately $1 \mathrm{hr}$. The liquid was decanted and analyzed for calcium, magnesium, strontium, and sodium by flame atomic absorption spectrometry on a Perkin-Elmer Model 5000 spectrophotometer. Replicate analyses yielded results within $2 \%$ for wt $\%$ calcium carbonate, $4 \%$ for strontium and sodium, and $8 \%$ for magnesium.

About 50 buffered acetic acid-insoluble residues were analyzed by X-ray diffraction (XRD). A third fraction of bulk sedi- ment was analyzed for carbon and oxygen isotopes on 112 selected samples at the University of Miami Rosenstiel School for Marine and Atmospheric Sciences on a Finnegan Mat-251 stable isotope ratio mass spectrometer. Isotopic values are reported as per mil $(\%)$ deviations from the PDB standard. Sample extractions were performed in an on-line automated device that reacts the sample with $100 \%$ phosphoric acid at $90^{\circ} \mathrm{C}$ for $20 \mathrm{~min}$. External error, determined by analyses of 20 replicate standards, is $\pm 0.026 \%$ for $\delta^{18} \mathrm{O}$ and $\pm 0.016 \%$ for $\delta^{13} \mathrm{C}$. In addition, selected bulk samples were studied by scanning electron microscopy on a JEOL T20 scanning electron microscope (SEM).

\section{RESULTS \\ Mineralogy}

Authigenic chalks become abundant below $70 \mathrm{mbsf}$, and authigenic celestite nodules appear commonly below 100 mbsf. Chalk is found in discrete layers or nodules (albeit not always obvious), but not in continually increasing abundance downcore. Much of the lower part of the core has a crusty texture that is intermediate between an ooze and a chalk.

Mineralogically, the uppermost sediment is a mixture of LMC, HMC, and aragonite (Fig. 2). Acid-insoluble residues are principally celestite, quartz, clay minerals, and dolomite. The buffered acetic acid leach used in the elemental analysis does not significantly dissolve dolomite, leaving it as an insoluble residue. Dolomite is present in such low quantities $(<1 \%)$ that it is not recognizable in XRD patterns of bulk samples. This finding is in contrast with studies of Bahamian periplatform sediments in which dolomite is often present in quantities as high as $15 \%$ of the fine fraction (Mullins et al., 1985b; Droxler et al., 1988). Of the 51 acid-insoluble residues analyzed, dolomite is present in $\mathbf{3 8}$. Dolomite is found as shallow as 26 mbsf and in intervals of high and low magnesium values.

The dominant mineralogy throughout the core consists of LMC. Aragonite and HMC have their greatest abundances in the upper part of the core. Furthermore, HMC is present only in minor quantities, with a maximum of only $10 \%$ in the total carbonates in the upper 1 mbsf (compared with $17 \%$ of the fine fraction; Droxler et al., this volume). Below $32 \mathrm{mbsf}(1.1 \mathrm{Ma})$, HMC is absent, and it is not found again in the lower sections. Aragonite is a significant component in the upper $60-70 \mathrm{~m}$ (1.9-2.3 Ma), varying from $15 \%$ to $40 \%$ of the total carbonates (the fine fraction in this interval ranges from $40 \%$ to $80 \%$ aragonite; Droxler et al., this volume). The aragonite content decreases to $<10 \%$ by $100 \mathrm{mbsf}(3 \mathrm{Ma})$, increases slightly to $\sim 15 \%$ at $130 \mathrm{mbsf}(4.25 \mathrm{Ma})$, and then decreases to $<6 \%$ until it finally disappears by $250 \mathrm{mbsf}(6 \mathrm{Ma})$. Eight discrete celestite nodules were identified, the shallowest appearing at $111.6 \mathrm{mbsf}$ (3.4 Ma). Celestite, apparently not occurring as discrete nodules, was found at 124.4,133.1,142.6, 177.7, 200, 206.7, and 219.3 mbsf.

\section{Minor Elements}

Results of elemental analyses are reported in Table 1. Calcium is reported as wt $\%$ calcium carbonate; all other elements are reported as ppm in calcium carbonate. Carbonate content varies from $85 \%$ to $98 \%$, increasing gradually downcore (Fig. 3). During the last $2 \mathrm{Ma}$ (about the upper $60 \mathrm{mbsf}$ ), the calcium carbonate content is related to sea-level cycles (Fig. 4). The wt $\%$ calcium carbonate is higher during highstands (interglacials), whereas the $\%$ coarse fraction and $\delta^{18} \mathrm{O}$ are lower (Droxler et al., this volume).

Strontium concentrations fluctuate considerably above 60 mbsf, with values ranging from 2200 to $4000 \mathrm{ppm}$ of the total carbonate fraction (Fig. 5). Over the next $40 \mathrm{~m}$, strontium concentrations gradually decline to approximately $1600 \mathrm{ppm}$, and 


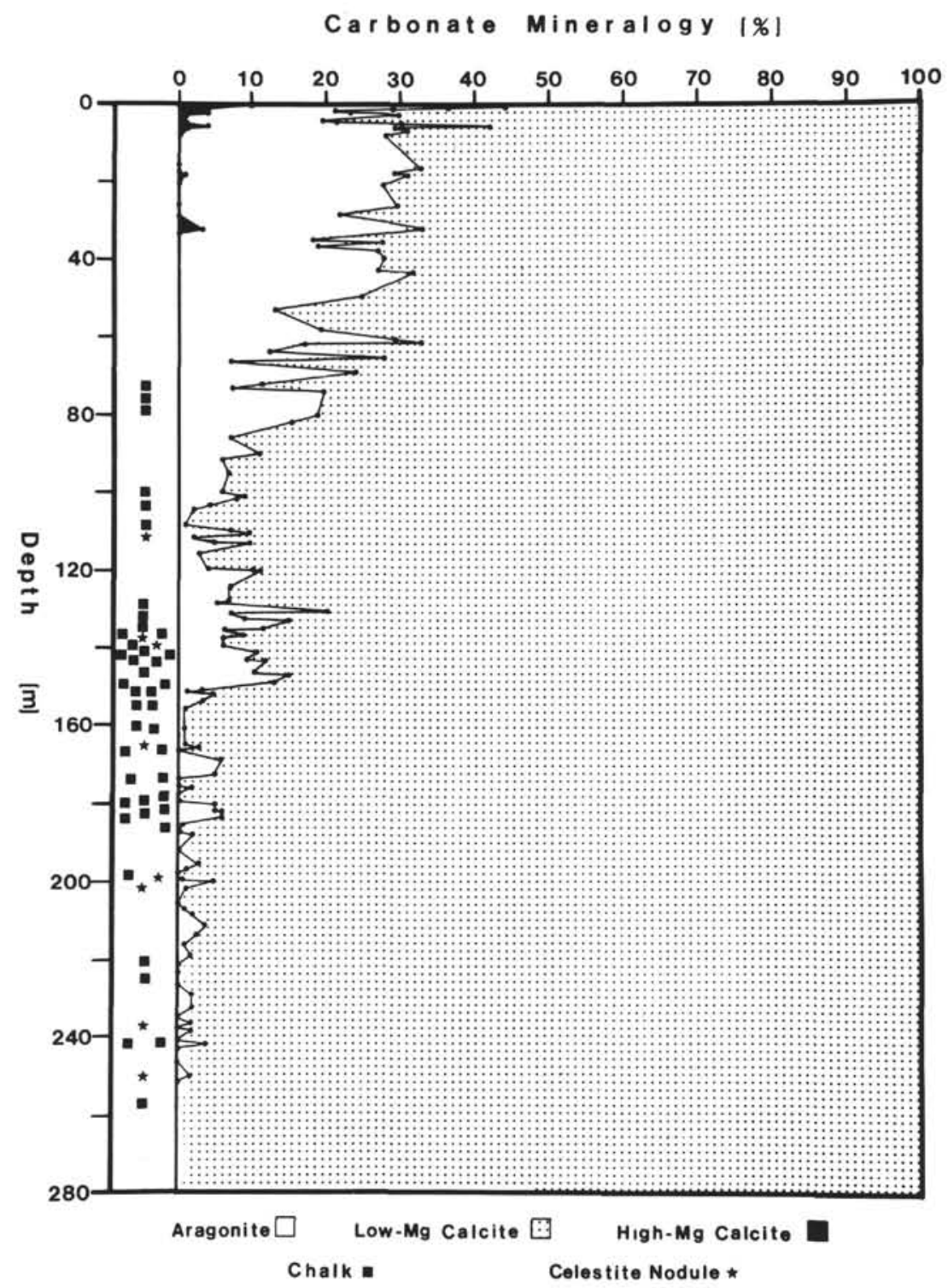

Figure 2. Relative $\%$ carbonate mineralogy. Solid squares $=$ chalks and stars $=$ celestite nodules.

they remain nearly constant throughout the rest of the core. Anomalously high strontium values below 100 mbsf are primarily caused by samples that contain small amounts of celestite (some celestite dissolves during dissolution). Milliman (1974) and, later, Boardman and Neumann (1984) have shown that bank-derived aragonite is more enriched in strontium than pelagic aragonite and calcite. The proportion of aragonite in the upper 60 mbsf implied by end-member mixing can be approximated by

$$
\begin{aligned}
(X)(7000 \mathrm{ppm})+(1-X)(1600 \mathrm{ppm})= & \text { observed ppm } \\
& (2200-4000),
\end{aligned}
$$

where $X=$ the proportion of high-Sr aragonite. End-member concentrations of strontium are taken to be $7000 \mathrm{ppm}$ in high-Sr aragonite and $1600 \mathrm{ppm}$ in low-Sr aragonite. This calculation suggests that high-Sr aragonite makes up $11 \%-44 \%$ of the bulk carbonate between 0 and $60 \mathrm{mbsf}$, which agrees well with percentages determined by XRD. We attribute the variability of strontium concentrations observed in the upper $60 \mathrm{~m}$ to glacialinterglacial cycles in periplatform-derived aragonite. Strontium concentrations and aragonite contents are higher during interglacial periods (see Droxler et al., this volume). Aragonite cycles have previously been documented in periplatform sediments (e.g., Kier and Pilkey, 1971; Droxler et al., 1983; Boardman and Neumann, 1984; Boardman et al., 1986; Droxler et al., 1988) and are being studied further by Droxler et al. (this volume). A plot of strontium in this interval (Fig. 6) shows that high strontium values also generally coincide with high calcium carbonate (Fig. 4). There are, however, occasional exceptions.

Strontium concentrations have previously been shown to be an important indicator of diagenesis in carbonate sediments. Biogenic calcium carbonate is enriched in strontium with respect to diagenetic LMC, the end product of marine burial diagenesis. During diagenesis, biogenic calcite (both high and low magnesium) and aragonite recrystallize to inorganic LMC, and the excess strontium is released into the pore waters (Baker et al., 1982). We ascribe the downcore decrease of strontium in the 
Table 1. Elemental and stable isotopic composition of bulk carbonate samples, Site 716.

\begin{tabular}{llllllll}
\hline $\begin{array}{r}\text { Core, section, } \\
\text { interval (cm) }\end{array}$ & $\begin{array}{l}\text { Depth } \\
(\mathrm{mbs})\end{array}$ & $\begin{array}{c}\mathrm{CaCO}_{3} \\
(\mathrm{wt} \%)\end{array}$ & $\begin{array}{c}\mathrm{Mg} \\
(\mathrm{ppm})\end{array}$ & $\begin{array}{c}\mathrm{Sr} \\
(\mathrm{ppm})\end{array}$ & $\begin{array}{c}\mathrm{Na} \\
(\mathrm{ppm})\end{array}$ & $\begin{array}{c}\delta^{13} \mathrm{C} \\
\left(\%_{0}\right)\end{array}$ & $\begin{array}{c}\delta^{18} \mathrm{O} \\
\left(\%_{0}\right)\end{array}$ \\
\hline $115-716 \mathrm{~B}-$
\end{tabular}

115-716B-

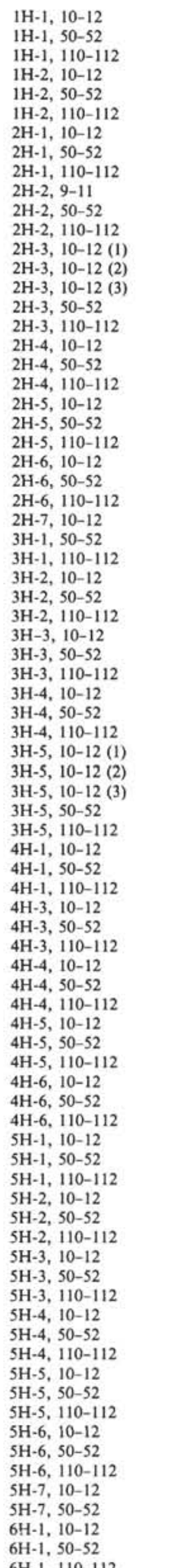

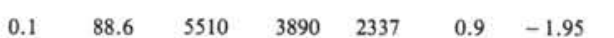

115-716B- (Cont.)

$\begin{array}{llllllll}0.1 & 88.6 & 5510 & 3890 & 2337 & 0.9 & -1.95 & 6 \\ 0.5 & 86.6 & 6341 & 3353 & 2419 & & & 6 \\ 1.1 & 87.2 & 4562 & 2357 & 2041 & & & 6 \\ 1.6 & 84.9 & 4571 & 2994 & 2002 & & & 6 \\ 2 & 87.3 & 5067 & 3376 & 2030 & 1.02 & -0.97 & 6 \\ 2.6 & 88.6 & 5138 & 3544 & 1882 & & & 6 \\ 4 & 86.4 & 2732 & 5107 & 1996 & & & 6 \\ 4.4 & 86.3 & 5274 & 2605 & 1996 & & & 6 \\ 5 & 88.9 & 4086 & 2466 & 1866 & 0.63 & -0.38 & 6 \\ 5.49 & 94.2 & 3420 & 3027 & 2521 & & & 6 \\ 5.9 & 92.2 & 4001 & 3336 & 2043 & & & 6 \\ 6.5 & 85.9 & 4537 & 2889 & 1932 & & & 6 \\ 7 & 93.1 & 4676 & 3958 & 2118 & 0.75 & -1.26 & 6 \\ 7 & 93.3 & 4909 & 3904 & 2112 & & & 6\end{array}$

$6 \mathrm{H}-2,10-12$ 6

$6 \mathrm{H}-3,10-12$

$6 \mathrm{H}-3,50-52$

$6 \mathrm{H}-3,110-112$

$6 \mathrm{H}-4,10-12$

$6 \mathrm{H}-4,110-112$

$6 \mathrm{H}-5,10-12$

$6 \mathrm{H}-5,110-112$

$6 \mathrm{H}-6,10-12$

$6 \mathrm{H}-6,110-112$

$6 \mathrm{H}-7,10-12$

$6 \mathrm{H}-7,50-52$

$7 \mathrm{H}-1,10-12$

$7 \mathrm{H}-1,50-52$

$7 \mathrm{H}-1,110-112$

$0.86-1.06$

$7 \mathrm{H}-2,10-12$

$7 \mathrm{H}-2,50-52$

$7 \mathrm{H}-2,110-112$

$7 \mathrm{H}-3,10-12$

$7 \mathrm{H}-3,110-112$

$0.74-1.21$

$7 \mathrm{H}-4,10-12$

$7 \mathrm{H}-4,50-52$

$7 \mathrm{H}-4,110-112$

$0.63-1.32$

$7 \mathrm{H}-5,10-12$

$7 \mathrm{H}-5,110-112$

$7 \mathrm{H}-6,10-12$

$0.44 \quad-0.67$

$7 \mathrm{H}-6,50-52$
$7 \mathrm{H}-6,110-112$

$7 \mathrm{H}-6,110-11$

$7 \mathrm{H}-7,10-12$

$8 \mathrm{H}-1,10-12$

$0.47-1.09$

$8 \mathrm{H}-1,50-52$

$8 \mathrm{H}-1,110-112$

$8 \mathrm{H}-2,10-12$

$8 \mathrm{H}-2,50-52$

$8 \mathrm{H}-2,110-112$

$\begin{array}{ll}0.75 & -0.8\end{array}$

$8 \mathrm{H}-3,10-12$

$8 \mathrm{H}-3,50-52$

$8 \mathrm{H}-3,110-112$

$\begin{array}{ll}0.32 & -2.28\end{array}$

$8 \mathrm{H}-4,10-12$

$8 \mathrm{H}-4,50-52$

$8 \mathrm{H}-4,110-112$

8 H-5, 10-12

$8 \mathrm{H}-5,50-52$

$8 \mathrm{H}-5,110-112$

$0.42-0.42$

$8 \mathrm{H}-6,10-12$ (1)

$8 \mathrm{H}-6,10-12$ (2)

$8 \mathrm{H}-6,10-12$ (3)

$8 \mathrm{H}-6,50-52$

8 H-6, 110-112

$0.85-1.28$

$8 \mathrm{H}-7,10-12$

9H-1, 10-12

9H-1, 50-52

$9 \mathrm{H}-1,110-112$

9H 2, 10-12

$$
\text { (CH) }
$$

9H 2, 50-52

$9 \mathrm{H}-2,110-112$

$9 \mathrm{H}-2,10-12$

(CH)

$9 \mathrm{H}-3,50-52$

$9 \mathrm{H}-3,110-112$

$9 \mathrm{H}-4,10-12$

$9 \mathrm{H}-4,50-52$

$9 \mathrm{H}-4,110-112$

$9 \mathrm{H}-5,10-12$

$9 \mathrm{H}-5,50-52$

(CH)

9 H-5, 110-112

$9 \mathrm{H}-6,10-12$

$9 \mathrm{H}-6,50-52$

$9 \mathrm{H}-6,110-112$

9H-7, 10-12

$0.64-0.25$

$6 \mathrm{H}-1,110-112$

$\begin{array}{lllll}43 & 91.1 & 3505 & 3221 & 1553\end{array}$

9H-7, 50-52 $\begin{array}{lllll}44.1 & 89.9 & 4656 & 3303 & 1530\end{array}$

$\begin{array}{lllll}44.5 & 91.5 & 4914 & 3314 & 2833\end{array}$

$\begin{array}{lllll}45.1 & 91.9 & 5228 & 3271 & 1811\end{array}$

$\begin{array}{lllll}45.6 & 91.5 & 5071 & 3006 & 1449\end{array}$

$\begin{array}{lllll}46 & 91.8 & 5358 & 2930 & 2006\end{array}$

$\begin{array}{lllll}46.6 & 93.2 & 4378 & 2505 & 1298\end{array}$

$\begin{array}{lllll}47.1 & 945 & 5053 & 3000 & 1298 \\ 47.5 & 899 & 4656 & 3425 & 159\end{array}$

$\begin{array}{lllll}48.1 & 914 & 4753 & 3341 & 1483\end{array}$

$\begin{array}{lllll}48.6 & 92.1 & 4714 & 3028 & 1481\end{array}$

$\begin{array}{lllll}49 & 90.4 & 5359 & 3177 & 3633 \\ 50.1 & 87.3 & 4345 & 3016 & 1517\end{array}$

$\begin{array}{lll}50.1 & 87.3 & 4345 \\ 50.5 & 86.2 & 5067\end{array}$

$\begin{array}{lllll}51.1 & 93.6 & 4129 & 2719 & 1523\end{array}$

$\begin{array}{lllll}51.6 & 88.7 & 4957 & 3267 & 1701\end{array}$

$\begin{array}{lllll}52 & 91.5 & 4802 & 3200 & 1750\end{array}$

$\begin{array}{lllll}52.2 & 86.6 & 5816 & 2737 & 1494\end{array}$

$\begin{array}{lllll}52.6 & 90.9 & 4824 & 2954 & 1471\end{array}$

$\begin{array}{lllll}53.2 & 92.3 & 3833 & 2741 & 1427\end{array}$

$\begin{array}{lllll}53.7 & 91.5 & 4385 & 3028 & 1341 \\ 54.1 & 91.3 & 4661 & 3028 & 1462\end{array}$

$\begin{array}{lllll}54.7 & 91.1 & 4166 & 2517 & 1407\end{array}$

$\begin{array}{lllll}55.2 & 93.2 & 4707 & 3074 & 1468\end{array}$

$\begin{array}{lllll}55.6 & 93.5 & 2596 & 3615 & 1937\end{array}$

$\begin{array}{lllll}56.2 & 90.7 & 3663 & 3151 & 1575\end{array}$

$\begin{array}{lllll}56.7 & 91.3 & 3352 & 3516 & 1586\end{array}$

$\begin{array}{lllll}57.1 & 89.8 & 3658 & 3647 & 3697\end{array}$

$\begin{array}{lllll}57.7 & 90.7 & 3843 & 2933 & 1483 \\ 58.2 & 93.8 & 4391 & 3378 & 1641\end{array}$

$\begin{array}{lllll}58.6 & 91.9 & 4985 & 3125 & 167\end{array}$

$\begin{array}{lllll}59.2 & 91.8 & 4320 & 3174 & 1649\end{array}$

$\begin{array}{lllll}59.7 & 94.7 & 3644 & 3235 & 1503\end{array}$

$\begin{array}{lllll}60.1 & 92.8 & 4242 & 3100 & 1636\end{array}$

$\begin{array}{lllll}60.7 & 92.8 & 3362 & 3770 & 1764 \\ 61.2 & 87.1 & 4111 & 3826 & 1625\end{array}$

$\begin{array}{lllll}61.2 & 87.1 & 4111 & 3826 & 162.5 \\ 61.6 & 87.1 & 4202 & 3398 & 1608\end{array}$

$\begin{array}{lllll}61.8 & 90.1 & 5105 & 2782 & 1175\end{array}$

$\begin{array}{lllll}62.2 & 91.5 & 4693 & 2741 & 123\end{array}$

$\begin{array}{lllll}62.8 & 92.6 & 4548 & 2933 & 1893\end{array}$

$\begin{array}{lllll}63.3 & 96.4 & 4229 & 2567 & 1023\end{array}$

$\begin{array}{lllll}63.7 & 90.6 & 3985 & 2786 & 1529\end{array}$

$\begin{array}{rrrrr}64.3 & 93.3 & 4989 & 2410 & 957 \\ 64.8 & 90.5 & 3930 & 2538 & 1120\end{array}$

$\begin{array}{lllll}65.2 & 91.7 & 3794 & 3418 & 1254\end{array}$

$\begin{array}{lllll}65.8 & 91.7 & 3698 & 2330 & 1070\end{array}$

$\begin{array}{lllll}66.3 & 93.6 & 5314 & 1840 & 957\end{array}$

$\begin{array}{lllll}66.7 & 93.3 & 3952 & 2789 & 1266\end{array}$

$\begin{array}{lllll}67.3 & 92.8 & 4108 & 2982 & 1094 \\ 67.8 & 92.6 & 4875 & 2644 & 1073\end{array}$

$\begin{array}{lllll}68.2 & 93.2 & 4489 & 2787 & 1085\end{array}$

$\begin{array}{lllll}68.8 & 91.9 & 3250 & 2719 & 1148\end{array}$

$\begin{array}{llllll}69.3 & 91.4 & 3849 & 2665 & 1092\end{array}$

$\begin{array}{lllll}69.3 & 93.8 & 3920 & 2649 & 1053\end{array}$

$\begin{array}{lllll}69.3 & 93.3 & 3617 & 2553 & 1096\end{array}$

$\begin{array}{lllll}69.7 & 95.6 & 2494 & 3030 & 1268\end{array}$

$\begin{array}{lllll}70.3 & 90.5 & 3036 & 2448 & 1101 \\ 70.8 & 93.9 & 3330 & 2296 & 1256\end{array}$

$\begin{array}{lllll}70.8 & 93.9 & 3330 & 2296 & 1256 \\ 71.2 & 94.3 & 2895 & 2136 & 1236\end{array}$

$\begin{array}{lllll}71.5 & 95.9 & 3381 & 2221 & 1154\end{array}$

$\begin{array}{lllll}71.9 & 95.1 & 5085 & 1750 & 893\end{array}$

$\begin{array}{llllr}71.9 & 95.1 & 5085 & 1750 & 893 \\ 73 & 93.7 & 3631 & 1989 & 1150 \\ 73 & 94.3 & 3581 & 1440 & 838\end{array}$

$\begin{array}{ll}0.48 & 0.24 \\ 0.89 & 0.8\end{array}$

$\begin{array}{lllll}73.4 & 93.9 & 3008 & 2839 & 1414\end{array}$

$\begin{array}{lllll}74 & 965 & 3546 & 1989 & 928\end{array}$

$\begin{array}{lll}75.5 & 922 & 4263\end{array}$

$1357 \quad 826$

$0.6 \quad 1.0$

$\begin{array}{lllll}74.9 & 90.6 & 4864 & 2050 & 1055\end{array}$

$\begin{array}{lllll}75.5 & 90.4 & 3897 & 2149 & \\ 76 & 96.9 & 2327 & 1861 & 1053\end{array}$

$\begin{array}{lllll}76.4 & 91.3 & 3011 & 1941 & 962\end{array}$

$\begin{array}{lllll}77 & 90.1 & 3177 & 3468 & 1709\end{array}$

$\begin{array}{rrrrr}77.5 & 93.7 & 3504 & 2628 & 1361 \\ 77.9 & 92.9 & 3702 & 1532 & 793\end{array}$

$0.63 \quad 0.86$

$\begin{array}{lllll}78.5 & 92.1 & 3233 & 2546 & 1243\end{array}$

$\begin{array}{lllll}79 & 87.3 & 3062 & 1568 & 867\end{array}$

$\begin{array}{lllll}79.4 & 91.6 & 2968 & 2882 & 1254\end{array}$

$\begin{array}{llllr}80 & 92.7 & 2793 & 2032 & 989\end{array}$
$0.13-0.79$

$0.31-0.64$

$0.99 \quad 0.11$

$0.96 \quad 0.03$

$\begin{array}{ll}1.03 & 0.37\end{array}$

$\begin{array}{ll}0.8 & 0.0\end{array}$

$\begin{array}{ll}1.02 & 0.08\end{array}$

$0.64-0.12$

$0.8 \quad 0.08$

$0.95 \quad 0.1$

$0.9 \quad 0.21$

$0.87 \quad 0.37$

$0.76-0.72$

$0.89 \quad 0.8$

$(2.040$

$\begin{array}{lllll}80.9 & 93.1 & 3143 & 2596 & 1094\end{array}$ 
Table 1 (continued).

\begin{tabular}{cccccccc}
\hline $\begin{array}{c}\text { Core, section, } \\
\text { interval }(\mathrm{cm})\end{array}$ & $\begin{array}{l}\text { Depth } \\
(\mathrm{mbsf})\end{array}$ & $\begin{array}{l}\mathrm{CaCO}_{3} \\
(\mathrm{wt} \%)\end{array}$ & $\begin{array}{c}\mathrm{Mg} \\
(\mathrm{ppm})\end{array}$ & $\begin{array}{c}\mathrm{Sr} \\
(\mathrm{ppm})\end{array}$ & $\begin{array}{c}\mathrm{Na} \\
(\mathrm{ppm})\end{array}$ & $\begin{array}{c}\delta^{13} \mathrm{C} \\
(\% 0)\end{array}$ & $\begin{array}{l}\delta^{18} \mathrm{O} \\
(\% 0)\end{array}$ \\
\hline
\end{tabular}

115-716B-(Cont.)

$10 \mathrm{H}-1,10-12$ $10 \mathrm{H}-1,50-52$

$10 \mathrm{H}-1,110-112$

$10 \mathrm{H}-2,10-12$

$10 \mathrm{H}-2,50-52$

$10 \mathrm{H}-2,110-112$

$10 \mathrm{H}-3,10-12$

$10 \mathrm{H}-3,50-52$

$10 \mathrm{H}-3,110-112$

$10 \mathrm{H}-4,10-12$

$10 \mathrm{H}-4,50-52$

$10 \mathrm{H}-5,10-12$

$10 \mathrm{H}-5,50-52$

$10 \mathrm{H}-5,110-112$

$10 \mathrm{H}-6,10-12$

$10 \mathrm{H}-6,50-52$

$10 \mathrm{H}-6,110-112$

$10 \mathrm{H}-7,10-12$

$11 \mathrm{H}-1,10-12$

$11 \mathrm{H}-1,50-52$

$11 \mathrm{H}-1,110-112$

$11 \mathrm{H}-2,10-12$

$11 \mathrm{H}-2,50-52$

$11 \mathrm{H}-2,110-112$

$11 \mathrm{H}-3,10-12$

$11 \mathrm{H}-3,110-112$

$11 \mathrm{H}-4,10-12$

$11 \mathrm{H}-4,50-52$

$11 \mathrm{H} .4,110-112$

$11 \mathrm{H}-5,10-12$

$11 \mathrm{H}-5,50-52$

$11 \mathrm{H}-6,10-12$

$11 \mathrm{H}-6,50-52$

$11 \mathrm{H}-6,110-112$

$11 \mathrm{H}-7,10-12$

$11 \mathrm{H}-7,50-52$

$12 \mathrm{H}-1,10-12$

$12 \mathrm{H}-1,50-52$

(CH)

$12 \mathrm{H}-1,110-112$

$12 \mathrm{H}-2,10-12$

$12 \mathrm{H}-2,110-112$
$12 \mathrm{H}-3,10-12$

$2 \mathrm{H}-3,10-12$
$(\mathrm{CH})$

(CH)
$2 \mathrm{H}-3,50-52$

$12 \mathrm{H}-3,110-112$

$12 \mathrm{H}-4,10-12$

$12 \mathrm{H}-4,50-52$

$12 \mathrm{H}-4,110-112$

$12 \mathrm{H}-5,10-12$

$12 \mathrm{H}-5,50-52$

$12 \mathrm{H}-5,110-112$

$12 \mathrm{H}-6,10-12$
$12 \mathrm{H}-6,50-52$

$12 \mathrm{H}-6,50-52$

$12 \mathrm{H}-6,110-112$ (CH)

$13 \mathrm{H}-1,10-12$

$13 \mathrm{H}-1,50-52$

$13 \mathrm{H}-1,110-112$

$13 \mathrm{H}-2,10-12\left(^{*}\right)$

$13 \mathrm{H}-2,50-52$

$13 \mathrm{H}-2,110-112$

$13 \mathrm{H}-3,10-12$

$13 \mathrm{H}-3,50-52$

$13 \mathrm{H}-3,110-112$

$13 \mathrm{H}-4,10-12$

$13 \mathrm{H}-4,50-52$
$13 \mathrm{H}-4,110-112$

$13 \mathrm{H}-5,10-12$

$13 \mathrm{H}-5,50-52$

$13 \mathrm{H}-5,110-112$

$13 \mathrm{H}-6,10-12$

$13 \mathrm{H}-6,50-52$
$13 \mathrm{H}-6,110-112$

$13 \mathrm{H}-7,10-12$

$13 \mathrm{H}-7,50-52$

$14 \mathrm{H}-1,20-22$ $\begin{array}{lllll}81.2 & 92.6 & 3368 & 2379 & 1146 \\ 81.6 & 93.6 & 2767 & 2596 & 1126\end{array}$

$\begin{array}{lllll}82.2 & 96.2 & 2386 & 2149 & 1062\end{array}$

$\begin{array}{lllll}82.7 & 92.2 & 2419 & 2499 & 1212 \\ 83.1 & 91.3 & 3331 & 2539 & 1088\end{array}$

$\begin{array}{lllll}83.7 & 84.6 & 2640 & 2236 & 1206\end{array}$

$\begin{array}{llll}84.2 & 87.4 & 3030 & 2007\end{array}$

$\begin{array}{lllll}84.6 & 94.1 & 2701 & 2115 & 921\end{array}$

$\begin{array}{lllll}85.2 & 93.1 & 2463 & 2101 & 1074\end{array}$

$\begin{array}{lllll}85.7 & 89.7 & 2253 & 2001 & 1066 \\ 86.1 & 96.4 & 3496 & 2400 & 1153\end{array}$

$\begin{array}{lllll}86.7 & 92.1 & 1652 & 2202 & 1254 \\ 87.2 & 93.3 & 1920 & 2362 & 1096\end{array}$

$\begin{array}{rrrrr}87.2 & 93.3 & 1920 & 2362 & 1096 \\ 87.6 & 93.7 & 2117 & 2245 & 957\end{array}$

$\begin{array}{lllll}88.2 & 93.6 & 1681 & 2266 & 1287\end{array}$

$\begin{array}{lllll}88.7 & 92.1 & 1823 & 2933 & 1459\end{array}$

$\begin{array}{lllll}89.1 & 94.1 & 1710 & 2391 & 1224 \\ 89.7 & 91.8 & 2195 & 2282 & 1070\end{array}$

$\begin{array}{lllll}90.2 & 93.6 & 2223 & 2149 & 1085\end{array}$

$\begin{array}{rrrrr}90.8 & 88.7 & 2359 & 1710 & 1062 \\ 91.2 & 91.9 & 2371 & 1837 & 872\end{array}$

91.8

92.3

92.7

93.3
93.8

94.2

94.8
95.3

95.7

96.3
96.8

96.8
97.2

97.8

98.3
98.7

98.7
99.3

99.3
99.8
100.2

100.4
100.8

101.4

101.9
102.3

102.9
103.4

103.8

104.4

104.9

105.3
105.9

105.9
106.4

106.8

107.4

107.9

108.3

108.9
109.4

110.1

110.1
110.5

111.1

112

112.6

113.1
113.5

113.5
114.1

114.6
115
115.6

115.6

116.1
116.5

117.1

117.6

118

118.6

119.1
119.5

119.9

$\begin{array}{llll}92.1 & 3078 & 2188 & 799\end{array}$

$\begin{array}{llll}91.3 & 2836 & 2880 & 1198\end{array}$

$\begin{array}{llll}93.1 & 3711 & 2263 & 901\end{array}$

$\begin{array}{llll}95.2 & 3340 & 2306 & 852\end{array}$

$\begin{array}{llll}91.2 & 3265 & 2198 & 742\end{array}$

$92.2 \quad 3030$

$\begin{array}{llll}96.5 & 2409 & 2112 & 982\end{array}$

$\begin{array}{llll}92.6 & 2746 & 2429 & 987\end{array}$

$\begin{array}{llll}92.8 & 2245 & 2266 & 1074\end{array}$

$\begin{array}{lll}2382 & 1953 & 963\end{array}$

$\begin{array}{llll}95.3 & 2405 & 1610 & 841\end{array}$

$\begin{array}{llll}93.4 & 2420 & 1676 & 915\end{array}$

$\begin{array}{llll}95.7 & 2130 & 1543 & 831\end{array}$

$\begin{array}{llll}95.3 & 2403 & 1838 & 827\end{array}$

$\begin{array}{llll}93.9 & 2464 & 1710 & 960 \\ 95.9 & 2493 & 1958 & 904\end{array}$

$\begin{array}{llll}95.7 & 2134 & 1850 & 1072\end{array}$

$\begin{array}{llll}95.1 & 2701 & 1764 & 1183\end{array}$

$95.1 \quad 2445 \quad 1811 \quad 911$

$\begin{array}{ll}93.8 & 3029\end{array}$

$\begin{array}{ll}96.5 & 2535 \\ 97.3 & 2767\end{array}$

$92.1-2768$

1662

1510
1406

855
798

$\begin{array}{rrrr}96.6 & 3002 & 1844 & 904 \\ 77.1 & 3511 & 11789 & 3743\end{array}$

$\begin{array}{llll}92.7 & 2574 & 1957 & 809\end{array}$

$\begin{array}{rrrr}91.9 & 2834 & 1935 & 80 \\ 90.4 & 3337 & 3475 & 1275\end{array}$

$\begin{array}{rrrr}90.4 & 3337 & 3475 & 1275 \\ 90.2 & 3287 & 1682 & 934\end{array}$

$\begin{array}{llll}91.9 & 3011 & 1800 & 1103\end{array}$

$\begin{array}{llll}92.1 & 3207 & 1711 & 1094\end{array}$

$\begin{array}{llll}93.1 & 2830 & 2245 & 1181\end{array}$

$\begin{array}{llll}94.7 & 2960 & 1377 & 939\end{array}$

$\begin{array}{rrrr}90.6 & 2567 & 2611 & 1621 \\ 90.3 & 3331 & 1852 & 835\end{array}$

$\begin{array}{rrrr}90.3 & 3331 & 1852 & 835 \\ 90.9 & 2616 & 1739 & 1068\end{array}$

$\begin{array}{rrrr}96.4 & 3058 & 1677 & 798\end{array}$

$\begin{array}{llll}95.8 & 2535 & 1704 & 1122\end{array}$

$\begin{array}{llll}95.7 & 2618 & 1797 & 956\end{array}$

$\begin{array}{llll}95.1 & 2487 & 1743 & 953 \\ 91.7 & 2524 & 1570 & 894\end{array}$

$\begin{array}{llll}97.3 & 2651 & 1803 & 1710\end{array}$
$0.21-0.88$

$0.44-0.1$

$0.36-1.6$

$0.27-1.47$

$0.48-1.0$

$0.49-0.48$

$0.19-1.27$

$\begin{array}{rrrr}94.3 & 2617 & 2322 & 1076 \\ 93.5 & 3050 & 2153 & 929\end{array}$

$\begin{array}{llll}95.9 & 2852 & 1683 & 712\end{array}$

$\begin{array}{ll}0.13 & -0.29\end{array}$

$0.21-0.04$

$1896 \quad 1031$

$\begin{array}{ll}0.47 & 0.27\end{array}$

$\begin{array}{ll}0.46 & -0.67\end{array}$ $\begin{array}{ll}0.08 & -0.37\end{array}$
Table 1 (continued).

$\begin{array}{llllllll}\text { Core, section, } & \text { Depth } & \mathrm{CaCO}_{3} & \mathrm{Mg} & \mathrm{Sr} & \mathrm{Na} & \delta^{13} \mathrm{C} & \delta^{18} \mathrm{O}\end{array}$

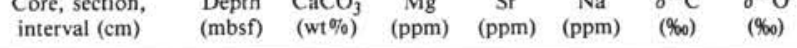

115-716B- (Cont.)

\begin{tabular}{|c|c|c|c|c|c|c|c|}
\hline $14 \mathrm{H}-1,70-72$ & 120.4 & 85.3 & 2991 & 2320 & 913 & 0.25 & -0.39 \\
\hline $14 \mathrm{H}-1,120-122(1)$ & 120.9 & 91.3 & 4097 & 1958 & 3390 & & \\
\hline $14 \mathrm{H}-1,120-122(2)$ & 120.9 & 91.4 & 3779 & 1936 & 801 & & \\
\hline $14 \mathrm{H}-1,120-122(3)$ & 120.9 & 91.5 & 3806 & 2018 & 801 & & \\
\hline $14 \mathrm{H}-1,120-122(4)$ & 120.9 & 93.4 & 3815 & 1986 & 777 & & \\
\hline $14 \mathrm{H}-2,70-72$ & 121.9 & 91.9 & 3063 & 1942 & 821 & & \\
\hline $14 \mathrm{H}-2,120-122$ & 122.4 & 93.1 & 2784 & 1862 & 826 & & \\
\hline $14 \mathrm{H}-3,20-22$ & 122.9 & 92.7 & 3618 & 1547 & 610 & & \\
\hline $14 \mathrm{H}-3,70-72$ & 123.4 & 92.5 & 3405 & 1619 & 680 & & \\
\hline $14 \mathrm{H}-3,120-122$ & 123.9 & 92.7 & 3083 & 2130 & 808 & & \\
\hline $14 \mathrm{H}-4,20-22$ & 124.4 & 90.9 & 3523 & 1764 & 1170 & 0.55 & 0.28 \\
\hline $14 \mathrm{H}-4,70-72$ & 124.9 & 89.7 & 3374 & 1742 & 785 & & \\
\hline $14 \mathrm{H}-4,120-122$ & 125.4 & 94.5 & 3366 & 1659 & 685 & & \\
\hline $14 \mathrm{H}-5,20-22$ & 125.9 & 94.7 & 3701 & 1731 & 2640 & & \\
\hline $14 \mathrm{H}-5,70-72$ & 126.4 & 96.4 & 3321 & 1862 & 1320 & & \\
\hline $14 \mathrm{H}-5,120-122$ & 126.9 & 93.4 & 3702 & 1597 & 658 & & \\
\hline $14 \mathrm{H}-6,20-22$ & 127.4 & 89.8 & 3653 & 1991 & 920 & & \\
\hline $14 \mathrm{H}-6,70-72$ & 127.9 & 91.4 & 3565 & 1684 & 735 & 0.68 & 0.57 \\
\hline $14 \mathrm{H}-6,120-122$ & 128.4 & 95.2 & 4082 & 1432 & 585 & 0.66 & 0.81 \\
\hline $14 \mathrm{H}-7,20-22$ & 128.9 & 95.5 & 3779 & 1469 & 638 & & \\
\hline $14 \mathrm{H}-7,70-72$ & 129.3 & 90.5 & 3842 & 1840 & 714 & & \\
\hline $15 \mathrm{H}-1,20-22$ & 129.5 & 98.7 & 3401 & 1561 & 857 & & \\
\hline $15 \mathrm{H}-1,70-72$ & 130 & 93.2 & 3478 & 1710 & 627 & & \\
\hline $15 \mathrm{H}-1,120-122$ & 130.5 & 95.1 & 2734 & 2634 & 1070 & & \\
\hline $\begin{array}{c}15 \mathrm{H}-2,20-22 \\
(\mathrm{CH})\end{array}$ & 131 & 93.5 & 3494 & 1694 & 712 & 0.83 & 0.9 \\
\hline $15 \mathrm{H}-2,70-72$ & 131.5 & 96.4 & 3423 & 2531 & 887 & & \\
\hline $15 \mathrm{H}-2,120-122$ & 132 & 95.1 & 3908 & 1975 & 1380 & & \\
\hline $\begin{array}{c}15 \mathrm{H}-3,20-22 \\
(\mathrm{CH})\end{array}$ & 132.5 & 95.9 & 3646 & 1781 & 695 & & \\
\hline $15 \mathrm{H}-3,70-72$ & 133 & 94.7 & 3492 & 2279 & 1040 & 0.61 & -0.28 \\
\hline $15 \mathrm{H}-3,120-122$ & 133.5 & 95.6 & 3630 & 1966 & 1020 & & \\
\hline $15 \mathrm{H}-4,20-22$ & 134 & 94.7 & 3695 & 2295 & 925 & & \\
\hline $15 \mathrm{H}-4,70-72$ & 134.5 & 93.9 & 3754 & 1954 & 733 & & \\
\hline $15 \mathrm{H}-4,120-122$ & 135 & 93.1 & 3715 & 2816 & 1170 & 0.79 & 0.05 \\
\hline $\begin{array}{c}15 \mathrm{H}-5,20-22 \\
(\mathrm{CH})\end{array}$ & 135.5 & 90.5 & 4347 & 1681 & 708 & & \\
\hline $15 \mathrm{H}-5,70-72$ & 136 & 94.8 & 3993 & 2172 & 897 & & \\
\hline $15 \mathrm{H}-5,120-122$ & 136.5 & 94.3 & 3859 & 2254 & 894 & & \\
\hline $\begin{array}{c}15 \mathrm{H}-6,20-22 \\
(\mathrm{CH})\end{array}$ & 137 & 88.1 & 4021 & 3293 & 1220 & & \\
\hline $\begin{array}{c}15 \mathrm{H}-6,27-30 \\
(\mathrm{CH})\end{array}$ & 137.07 & 94.3 & 4209 & 1623 & 1940 & 0.75 & 0.78 \\
\hline $15 \mathrm{H}-6,40-42\left(^{*}\right)$ & 137.2 & 34.1 & 3916 & 58153 & 2240 & & \\
\hline $15 \mathrm{H}-6,70-72$ & 137.5 & 93.1 & 3831 & 1926 & 723 & & \\
\hline $15 \mathrm{H}-6,120-122$ & 138 & 93.6 & 4025 & 1809 & 707 & & \\
\hline $15 \mathrm{H}-7,20-22$ & 138.5 & 91.7 & 4205 & 1779 & 974 & & \\
\hline $16 \mathrm{H}-1,20-22$ & 139.1 & 94.5 & 3809 & 1659 & 716 & & \\
\hline $16 \mathrm{H}-1,58-60$ & 139.48 & 93.7 & 3797 & 1513 & 1190 & 0.8 & 0.7 \\
\hline
\end{tabular}

(CH)

$\begin{array}{llllll}16 \mathrm{H}-1,70-72 & 139.6 & 94.6 & 3809 & 2276 & 643\end{array}$

$\begin{array}{llllll}16 \mathrm{H}-1,120-122 & 140.1 & 96.2 & 4020 & 1621 & 700\end{array}$

$\begin{array}{llllll}16 \mathrm{H}-2,20-22 & 140.6 & 95.1 & 4036 & 1795 & 690\end{array}$

$\begin{array}{llllrr}16 \mathrm{H}-2,70-72\left(^{*}\right) & 141.1 & 42.6 & 3465 & 41793 & 1730 \\ & 141.6 & 97.9 & 3426 & 1885 & 816\end{array}$

$\begin{array}{llllll}16 \mathrm{H}-3,20-22 & 142.1 & 93.5 & 3704 & 1649 & 664\end{array}$

(CH)

$16 \mathrm{H}-3,57-59$

(CH)

$16 \mathrm{H}-3,70-7$
$(\mathrm{CH})$

$16 \mathrm{H}-3,107-109$ (CH)

$16 \mathrm{H}-3,120-122$ (CH)

$16 \mathrm{H}-4,70-72$

$16 \mathrm{H}-4,120-122$

$16 \mathrm{H}-5,20-22$

$16 \mathrm{H}-5,70-72$

$16 \mathrm{H}-5,120-122$

$6 \mathrm{H}-6,16$

$16 \mathrm{H}-6,20-22$

$16 \mathrm{H}-6,70-72$

$16 \mathrm{H}-6,120-122$

$16 \mathrm{H}-7,20-22$

(CH)
$17 \mathrm{H}-1,20-22$

$17 \mathrm{H}-1,70-72$

$17 \mathrm{H}-1,120-122$

142.47

$95.9 \quad 3878$

1568

967

$\begin{array}{ll}0.85 & 0.78\end{array}$

$17 \mathrm{H}-2,20-22$

$\begin{array}{lrrrr}142.6 & 91.2 & 3922 & 3050 & 669 \\ 142.97 & 93.2 & 4301 & 1492 & 2140\end{array}$

$\begin{array}{lllll}143.1 & 93.6 & 4068 & 1584 & 1170\end{array}$

$\begin{array}{lllll}1441 & 968 & 3922 & 1817 & 818\end{array}$

$\begin{array}{lllll}144.6 & 95.7 & 3993 & 1966 & 736\end{array}$

$\begin{array}{lllll}145.1 & 93.5 & 4046 & 1593 & 765\end{array}$

$\begin{array}{lllll}145.6 & 94.3 & 4225 & 1960 & 706\end{array}$

$\begin{array}{llllr}146.1 & 96.7 & 4236 & 1760 & 669 \\ 146.56 & 96.4 & 4797 & 1565 & 1400\end{array}$

$\begin{array}{lllll}146.6 & 96.9 & 4253 & 1711 & 664\end{array}$

$\begin{array}{lllll}147.1 & 94.8 & 4275 & 2013 & 786\end{array}$

$\begin{array}{lllll}147.6 & 90.1 & 4231 & 1720 & 774\end{array}$

$\begin{array}{lllll}148.1 & 93.5 & 3829 & 2156 & 844 \\ 148.6 & 88.1 & 4186 & 1962 & 751\end{array}$

$\begin{array}{lllll}148.8 & 93.4 & 3855 & 1874 & 765\end{array}$

$\begin{array}{lllll}149.3 & 93.2 & 4200 & 1651 & 658\end{array}$

$\begin{array}{rrrrr}149.8 & 95.6 & 3507 & 2216 & 1100 \\ 150.3 & 97.9 & 3682 & 1706 & 781\end{array}$ 
Table 1 (continued).

\begin{tabular}{|c|c|c|c|c|c|c|c|}
\hline $\begin{array}{l}\text { Core, section, } \\
\text { interval }(\mathrm{cm})\end{array}$ & $\begin{array}{l}\text { Depth } \\
\text { (mbsf) }\end{array}$ & $\begin{array}{l}\mathrm{CaCO}_{3} \\
(\mathrm{wt} \%)\end{array}$ & $\underset{(\mathrm{ppm})}{\mathrm{Mg}}$ & $\underset{(\mathrm{ppm})}{\mathrm{Sr}}$ & $\underset{(\mathrm{ppm})}{\mathrm{Na}}$ & $\begin{array}{l}\delta^{13} \mathrm{C} \\
(\% 00)\end{array}$ & $\begin{array}{l}\delta^{18} \mathrm{O} \\
\left(\%_{00}\right)\end{array}$ \\
\hline \multicolumn{8}{|l|}{ 115-716B-(Cont.) } \\
\hline $17 \mathrm{H}-2,70-72$ & 150.8 & 95.1 & 3659 & 2357 & 936 & & \\
\hline $\begin{array}{c}17 \mathrm{H}-2,106-107 \\
(\mathrm{CH})\end{array}$ & 151.16 & 96.1 & 4725 & 1469 & 998 & & \\
\hline $\begin{array}{c}17 \mathrm{H}-2,120-122 \\
(\mathrm{CH})\end{array}$ & 151.3 & 94.7 & 4736 & 1364 & 653 & & \\
\hline $\begin{array}{c}17 \mathrm{H}-3,20-22 \\
(\mathrm{CH})\end{array}$ & 151.8 & 94.1 & 4646 & 1434 & 573 & & \\
\hline $\begin{array}{c}17 \mathrm{H}-3,36-38 \\
(\mathrm{CH})\end{array}$ & 151.96 & 94.4 & 4689 & 1412 & 961 & -0.6 & 1.13 \\
\hline $17 \mathrm{H}-3,70-72$ & 152.3 & 95.5 & 4320 & 1535 & 529 & & \\
\hline $17 \mathrm{H}-3,120-122$ & 152.8 & 97.1 & 3708 & 1613 & 612 & & \\
\hline $17 \mathrm{H}-4,20-22$ & 153.3 & 98.1 & 4014 & 1399 & 521 & & \\
\hline $17 \mathrm{H}-4,70-72$ & 153.8 & 98.4 & 3989 & 1343 & 562 & & \\
\hline $17 \mathrm{H}-4,120-122$ & 154.3 & 94.4 & 4147 & 1476 & 743 & 0.38 & 0.94 \\
\hline $17 \mathrm{H}-5,20-22$ & 154.8 & 91.5 & 4569 & 1996 & 993 & & \\
\hline $17 \mathrm{H}-5,70-72$ & 155.3 & 95.5 & 4929 & 1432 & 596 & & \\
\hline $\begin{array}{c}17 \mathrm{H}-5,120-122 \\
(\mathrm{CH})\end{array}$ & 155.8 & 94.2 & 3603 & 1322 & 664 & & \\
\hline $\begin{array}{c}17 \mathrm{H}-6,20-22 \\
(\mathrm{CH})\end{array}$ & 156.3 & 97.5 & 3264 & 1476 & 756 & 0.6 & 0.7 \\
\hline $17 \mathrm{H}-6,70-72$ & 156.8 & 96.9 & 3156 & 1762 & 863 & & \\
\hline $17 \mathrm{H}-6,120-122$ & 157.3 & 94.7 & 3288 & 1531 & 711 & & \\
\hline $17 \mathrm{H}-7,20-22$ & 157.8 & 95.9 & 3137 & 1613 & 633 & & \\
\hline $18 \mathrm{H}-1,20-22$ & 158.5 & 94.9 & 3314 & 1401 & 516 & & \\
\hline $18 \mathrm{H}-1,70-72$ & 159 & 92.8 & 3534 & 1559 & 648 & & \\
\hline $18 \mathrm{H}-1,120-122$ & 159.5 & 95.7 & 3178 & 1605 & 544 & & \\
\hline $18 \mathrm{H}-2,20-22$ & 160 & 96.9 & 3139 & 1662 & 546 & & \\
\hline $18 \mathrm{H}-2,70-72$ (1) & 160.5 & 96.1 & 3195 & 1520 & 633 & & \\
\hline $18 \mathrm{H}-2,70-72$ (2) & 160.5 & 96.8 & 3114 & 1508 & 587 & & \\
\hline $18 \mathrm{H}-2,70-72(3)$ & 160.5 & 97.6 & 3155 & 1532 & 582 & & \\
\hline $18 \mathrm{H}-2,70-72$ (4) & 160.5 & 94.7 & 3239 & 1556 & 605 & & \\
\hline $18 \mathrm{H}-2,120-122$ & 161 & 93.9 & 3238 & 1467 & 503 & & \\
\hline $\begin{array}{c}18 \mathrm{H}-3,20-22 \\
(\mathrm{CH})\end{array}$ & 161.5 & 95.5 & 3573 & 1421 & 550 & 0.56 & 0.92 \\
\hline $\begin{array}{c}18 \mathrm{H}-3,70-72 \\
(\mathrm{CH})\end{array}$ & 162 & 95.7 & 3779 & 1375 & 810 & 0.52 & 1.0 \\
\hline $18 \mathrm{H}-3,120-122$ & 162.5 & 95.8 & 3480 & 1421 & 477 & & \\
\hline $18 \mathrm{H}-4,20-22$ & 163 & 92.8 & 3774 & 1445 & 503 & & \\
\hline $18 \mathrm{H}-4,70-72$ & 163.5 & 94.5 & 4105 & 1354 & 510 & & \\
\hline $18 \mathrm{H}-4,120-122$ & 164 & 94.9 & 3711 & 1411 & 502 & & \\
\hline $18 \mathrm{H}-5,20-22$ & 164.5 & 94.9 & 3769 & 1923 & 596 & & \\
\hline $18 \mathrm{H}-5,70-72$ & 165 & 942 & 3590 & 1397 & 499 & 0.62 & 1.03 \\
\hline $18 \mathrm{H}-5,120-122$ & $165-5$ & 94.2 & 3685 & 1476 & 531 & & \\
\hline $18 \mathrm{H}-6,8-11\left(^{*}\right)$ & 165.88 & 30.9 & 2819 & 69218 & 2210 & & \\
\hline $18 \mathrm{H}-6,20-22$ & 166 & 94.8 & 3287 & 1781 & 716 & & \\
\hline $\begin{array}{c}18 \mathrm{H}-6,70-72 \\
(\mathrm{CH})\end{array}$ & 166.5 & 98.6 & 3544 & 1302 & 388 & & \\
\hline $18 \mathrm{H}-6,120-122$ & 167 & 91.9 & 3507 & 1618 & 421 & 0.53 & 1.29 \\
\hline $\begin{array}{c}18 \mathrm{H}-7,20-22 \\
(\mathrm{CH})\end{array}$ & 167.5 & 96.4 & 3278 & 1333 & 463 & & \\
\hline $19 \mathrm{H}-1,20-22$ & 168.1 & 95.4 & 4108 & 1490 & 512 & & \\
\hline $19 \mathrm{H}-1,70-72$ & 168.6 & 94.1 & 3872 & 1839 & 827 & & \\
\hline $19 \mathrm{H}-1,120-122$ & 169.1 & 95.5 & 3856 & 1810 & 778 & 0.16 & 0.08 \\
\hline $19 \mathrm{H}-2,20-22$ & 169.6 & 97.4 & 3412 & 1565 & 1630 & & \\
\hline $19 \mathrm{H}-2,71-72$ & 170.11 & 95.2 & 3330 & 1589 & 1540 & & \\
\hline $19 \mathrm{H}-2,120-122$ & 170.6 & 98.6 & 3084 & 1645 & 770 & & \\
\hline $19 \mathrm{H}-3,20-22$ & 171.1 & 96.6 & 3304 & 1343 & 1240 & & \\
\hline $19 \mathrm{H}-3,70-72$ & 171.6 & 98.5 & 3401 & 1541 & 1780 & & \\
\hline $19 \mathrm{H}-3,120-122$ & 172.1 & 97.5 & 3626 & 1399 & 1530 & & \\
\hline $\begin{array}{c}19 \mathrm{H}-4,20-22 \\
(\mathrm{CH})\end{array}$ & 172.6 & 90.4 & 3722 & 3219 & 542 & -0.03 & 0.61 \\
\hline $19 \mathrm{H}-4,70-72$ & 173.1 & 92.5 & 3533 & 1649 & 990 & & \\
\hline $\begin{array}{c}19 \mathrm{H}-4,120-122 \\
(\mathrm{CH})\end{array}$ & 173.6 & 95.4 & 3272 & 1411 & 784 & 0.15 & 0.61 \\
\hline $19 \mathrm{H}-5,20-22$ & 174.1 & 95.7 & 3011 & 1432 & 585 & & \\
\hline $19 \mathrm{H}-5,70-72$ & 174.6 & 93.5 & 2890 & 1424 & 439 & & \\
\hline $19 \mathrm{H}-5,120-122$ & 175.1 & 96.1 & 2920 & 1421 & 467 & & \\
\hline $19 \mathrm{H}-6,20-22$ & 175.6 & 96.7 & 2829 & 1459 & 478 & & \\
\hline $\begin{array}{c}19 \mathrm{H}-6,70-72 \\
(\mathrm{CH})\end{array}$ & 176.1 & 92.8 & 3072 & 3420 & 610 & & \\
\hline $19 \mathrm{H}-6,120-122$ & 176.6 & 95.3 & 2998 & 1501 & 643 & & \\
\hline $\begin{array}{c}19 \mathrm{H}-7,20-22 \\
(\mathrm{CH})\end{array}$ & 177.1 & 95.7 & 3152 & 1354 & 533 & 0.25 & 0.85 \\
\hline $20 \mathrm{H}-1,20-22$ & 177.7 & 91.3 & 3079 & 2655 & 609 & & \\
\hline $20 \mathrm{H}-1,70-72$ & 178.2 & 97.8 & 2865 & 1435 & 552 & & \\
\hline $20 \mathrm{H}-1,120-122$ & 178.7 & 96.3 & 2805 & 1508 & 664 & & \\
\hline $\begin{array}{c}20 \mathrm{H}-2,20-22 \\
(\mathrm{CH})\end{array}$ & 179.2 & 95.6 & 3003 & 1400 & 498 & & \\
\hline $\begin{array}{c}20 \mathrm{H}-2,70-72 \\
(\mathrm{CH})\end{array}$ & 179.7 & 97.9 & 3090 & 1379 & 541 & 0.1 & 0.7 \\
\hline $\begin{array}{c}20 \mathrm{H}-2,120-122 \\
(\mathrm{CH})\end{array}$ & 180.12 & 94.7 & 3330 & 1375 & 606 & & \\
\hline
\end{tabular}

Table 1 (continued).

\begin{tabular}{|c|c|c|c|c|c|c|c|}
\hline $\begin{array}{l}\text { Core, section, } \\
\text { interval }(\mathrm{cm})\end{array}$ & $\begin{array}{l}\text { Depth } \\
\text { (mbsf) }\end{array}$ & $\begin{array}{l}\mathrm{CaCO}_{3} \\
(\mathrm{wt} \%)\end{array}$ & $\underset{(\mathrm{ppm})}{\mathrm{Mg}}$ & $\underset{(\mathrm{ppm})}{\mathrm{Sr}}$ & $\underset{(\mathrm{ppm})}{\mathrm{Na}}$ & $\begin{array}{l}\delta^{13} \mathrm{C} \\
(\% 0)\end{array}$ & $\begin{array}{l}\delta^{18} \mathrm{O} \\
\left(\%\left(\omega_{0}\right)\right.\end{array}$ \\
\hline \multicolumn{8}{|l|}{ 115-716B-(Cont.) } \\
\hline $20 \mathrm{H}-3,20-22$ & 180.7 & 94.7 & 3032 & 2294 & 1320 & & \\
\hline $20 \mathrm{H}-3,70-72$ & 181.2 & 95.2 & 3507 & 1234 & 862 & & \\
\hline $\begin{array}{c}20 \mathrm{H}-3,120-122 \\
(\mathrm{CH})\end{array}$ & 181.7 & 95.1 & 3314 & 1522 & 748 & & \\
\hline $\begin{array}{c}20 \mathrm{H}-4,20-22 \\
(\mathrm{CH})\end{array}$ & 182.2 & 93.3 & 3393 & 1736 & 1140 & -0.07 & 0.05 \\
\hline $\begin{array}{c}20 \mathrm{H}-4,120-122 \\
(\mathrm{CH})\end{array}$ & 183.2 & 97.2 & 3114 & 1837 & 751 & & \\
\hline $20 \mathrm{H}-5,20-22$ & 183.7 & 96.3 & 3428 & 1354 & 669 & & \\
\hline $20 \mathrm{H}-5,70-72$ & 184.2 & 95.8 & 2787 & 1559 & 795 & & \\
\hline $20 \mathrm{H}-5,120-122$ & 184.7 & 96.4 & 2846 & 1523 & 566 & & \\
\hline $20 \mathrm{H}-6,20-22$ & 185.2 & 97.2 & 2712 & 1467 & 772 & 0.34 & 0.43 \\
\hline $20 \mathrm{H}-6,70-72$ & 185.7 & 94.4 & 2894 & 1572 & 834 & & \\
\hline $20 \mathrm{H}-6,120-122$ & 186.2 & 97.7 & 2894 & 1769 & 517 & & \\
\hline $20 \mathrm{H}-7,20-22$ & 186.7 & 98.7 & 2255 & 1389 & 689 & & \\
\hline $\begin{array}{c}20 \mathrm{H}-7,70-72 \\
(\mathrm{CH})\end{array}$ & 187.2 & 95.9 & 3026 & 1688 & 460 & 0.03 & 0.52 \\
\hline $21 \mathrm{H}-1,20-22$ & 187.25 & 96.8 & 2645 & 1353 & 587 & 0.09 & -0.47 \\
\hline $2 \mathrm{HH}-1,70-72$ & 187.75 & 97.9 & 2477 & 1573 & 746 & & \\
\hline $21 \mathrm{H}-1,120-122$ & 188.25 & 95.1 & 2740 & 1478 & 515 & & \\
\hline $21 \mathrm{H}-2,20-22$ & 188.75 & 97.4 & 2083 & 1435 & 613 & & \\
\hline $21 \mathrm{H}-2,70-72$ & 189.25 & 96.5 & 2681 & 1657 & 556 & & \\
\hline $21 \mathrm{H}-2,120-122$ & 189.75 & 948 & 2698 & 1614 & 616 & & \\
\hline $21 \mathrm{H}-3,20-22$ & 190.25 & $98-4$ & 2590 & 1637 & & & \\
\hline $21 \mathrm{H}-3,70-72$ & 190.75 & 95.7 & 2513 & 2018 & 985 & & \\
\hline $21 \mathrm{H}-3,120-122$ & 191.25 & 95.4 & 3008 & 2055 & 421 & 0.03 & 0.45 \\
\hline $21 \mathrm{H}-4,20-22$ & 191.75 & 91.7 & 2921 & 1344 & 544 & & \\
\hline $21 \mathrm{H}-4,70-72$ & 192.25 & 94.7 & 2561 & 1667 & 716 & & \\
\hline $21 \mathrm{H}-4,120-122$ (1) & 192.75 & 97.4 & 2446 & 1496 & 552 & & \\
\hline $21 \mathrm{H}-4,120-122(2)$ & 192.75 & 98.6 & 2436 & 1476 & 592 & & \\
\hline $21 \mathrm{H}-4,120-122(3)$ & 192.75 & 97.9 & 2477 & 1553 & 572 & & \\
\hline $21 \mathrm{H}-4,120-122$ (4) & 192.75 & 96.6 & 2609 & 1556 & 570 & & \\
\hline $21 \mathrm{H}-5,20-22$ & 193.25 & 97.9 & 2528 & 1246 & 449 & 0.01 & 0.26 \\
\hline $21 \mathrm{H}-5,70-72$ & 193.75 & 98.1 & 2406 & 1323 & 521 & & \\
\hline $21 \mathrm{H}-5,120-122$ & 194.25 & 97.2 & 2676 & 1359 & 552 & & \\
\hline $21 \mathrm{H}-6,20-22$ & 194.75 & 95.7 & 2723 & 1411 & 732 & & \\
\hline $21 \mathrm{H}-6,70-72$ & 195.25 & 94.2 & 2553 & 1729 & 707 & & \\
\hline $21 \mathrm{H}-6,120-122$ & 195.75 & 93.2 & 2741 & 1581 & 788 & 0.23 & 0.29 \\
\hline $21 \mathrm{H}-7,20-22$ & 196.25 & 96.2 & 2739 & 1688 & 763 & & \\
\hline $22 \mathrm{H}-1,20-22$ & 197 & 88.6 & 2829 & 1878 & 607 & & \\
\hline $22 \mathrm{H}-1,70-72$ & 197.5 & 91.2 & 2901 & 1541 & 823 & & \\
\hline $22 \mathrm{H}-1,120-122$ & 198 & 93.1 & 2929 & 1488 & 567 & & \\
\hline $\begin{array}{c}22 \mathrm{H}-2,20-22 \\
(\mathrm{CH})\end{array}$ & 198.5 & 93.4 & 3169 & 1171 & 739 & 0.1 & 0.93 \\
\hline $22 \mathrm{H}-2,70-72$ & 199 & 93.6 & 3083 & 1408 & 1040 & & \\
\hline $22 \mathrm{H}-2,120-122$ & 199.5 & 94.5 & 2602 & 2034 & 961 & & \\
\hline $22 \mathrm{H}-3,10-12\left(^{*}\right)$ & 199.9 & 48.9 & 2406 & 17644 & 2640 & & \\
\hline $22 \mathrm{H}-3,20-22$ & 200 & 95.9 & 2351 & 2107 & 550 & -0.07 & -0.07 \\
\hline $22 \mathrm{H}-3,70-72$ & 200.5 & 90.4 & 2742 & 1750 & 415 & & \\
\hline $22 \mathrm{H}-3,120-122$ & 201 & 93.4 & 2553 & 1649 & 771 & & \\
\hline $22 \mathrm{H}-4,20-22$ & 201.5 & 93.2 & 2782 & 1651 & 1010 & & \\
\hline $22 \mathrm{H}-4,66-68\left(^{*}\right)$ & 201.96 & 35.3 & 2413 & 55593 & 2370 & & \\
\hline $22 \mathrm{H}-4,70-72$ & 202 & 93.5 & 2461 & 1500 & 529 & 0.21 & 0.19 \\
\hline $22 \mathrm{H}-4,120-122$ & 202.5 & 91.8 & 2531 & 1538 & 799 & & \\
\hline $22 \mathrm{H}-5,20-22$ & 203 & 94.5 & 2444 & 1623 & 817 & & \\
\hline $22 \mathrm{H}-5,70-72$ & 203.5 & 96.7 & 2478 & 1688 & 957 & & \\
\hline $22 \mathrm{H}-5,120-122$ & 204 & 92.9 & 2564 & 1649 & 760 & & \\
\hline $22 \mathrm{H}-6,20-22$ & 204.5 & 94.4 & 2399 & 1960 & 1010 & & \\
\hline $22 \mathrm{H}-6,70-72$ & 205 & 91.8 & 2227 & 2243 & 1230 & & \\
\hline $22 \mathrm{H}-6,120-122$ & 205.5 & 91.7 & 2457 & 1667 & 1180 & 0.09 & -0.29 \\
\hline $22 \mathrm{H}-7,20-22$ & 206 & 93.7 & 2501 & 1614 & 1150 & & \\
\hline $22 \mathrm{H}-7,70-72$ & 206.5 & 91.2 & 2583 & 1607 & 839 & & \\
\hline $23 \mathrm{H}-1,20-22$ & 206.7 & 84.5 & 2524 & 5983 & 1240 & 0.13 & -0.31 \\
\hline $23 \mathrm{H}-1,70-72$ & 207.2 & 94.3 & 2581 & 1580 & 911 & & \\
\hline $23 \mathrm{H}-1,120-122$ & 207.7 & 94.8 & 2544 & 1556 & 1010 & & \\
\hline $23 \mathrm{H}-2,20-22$ & 208.2 & 95.5 & 2593 & 1613 & 949 & 0.18 & -0.03 \\
\hline $23 \mathrm{H}-2,70-72$ & 208.7 & 94.2 & 2756 & 1614 & 823 & & \\
\hline $23 \mathrm{H}-2,120-122$ & 209.2 & 95.3 & 2629 & 1626 & 1010 & & \\
\hline $23 \mathrm{H}-3,20-22$ & 209.7 & 95.1 & 2504 & 1788 & 1060 & & \\
\hline $23 \mathrm{H}-3,70-72$ & 210.2 & 93.4 & 2371 & 1488 & 926 & & \\
\hline $23 \mathrm{H}-3,120-122$ & 210.7 & 91.8 & 2730 & 1619 & 944 & & \\
\hline $23 \mathrm{H}-4,20-22$ & 211.2 & 92.9 & 2666 & 1649 & 1240 & & \\
\hline $23 \mathrm{H}-4,70-72$ & 211.7 & 94.2 & 2421 & 1896 & 1120 & 0.12 & -0.83 \\
\hline $23 \mathrm{H}-4,120-122$ & 212.2 & 90.8 & 2529 & 1854 & 1310 & & \\
\hline $23 \mathrm{H}-5,70-72$ & 213.2 & 95.4 & 2038 & 1788 & 1320 & & \\
\hline $23 \mathrm{H}-5,120-122$ & 213.7 & 93.4 & 2061 & 1649 & 1040 & & \\
\hline $23 \mathrm{H}-6,20-22$ & 214.2 & 93.7 & 1975 & 1556 & 1160 & & \\
\hline $23 \mathrm{H}-6,70-72$ & 214.7 & 93.9 & 1964 & 1649 & 1110 & 0.06 & -0.76 \\
\hline $23 \mathrm{H}-6,120-122$ (1) & 215.2 & 95.1 & 2012 & 1667 & 1220 & & \\
\hline $23 \mathrm{H}-6,120-122$ (2) & 215.2 & 94.8 & 2012 & 1667 & 1260 & & \\
\hline $23 \mathrm{H}-6,120-122(3)$ & 215.2 & 94.9 & 2018 & 1659 & 1250 & & \\
\hline $23 \mathrm{H}-6,120-122$ (4) & 215.2 & 96.9 & 2007 & 1662 & 1210 & & \\
\hline
\end{tabular}


Table 1 (continued).

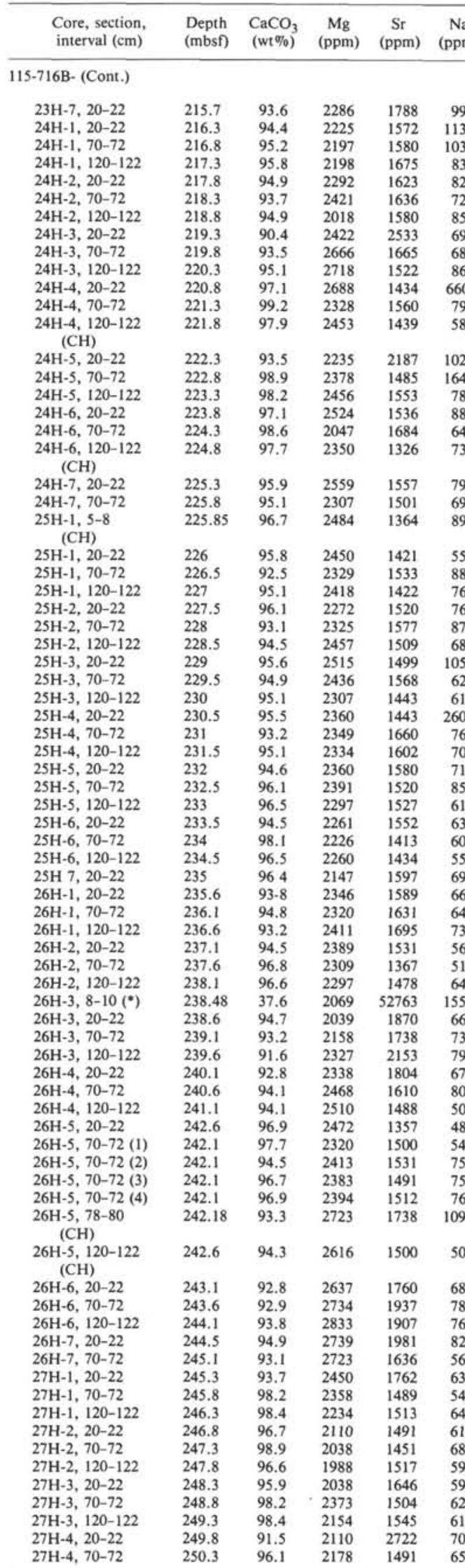

Table 1 (continued).

\begin{tabular}{|c|c|c|c|c|c|c|c|}
\hline $\begin{array}{l}\text { Core, section, } \\
\text { interval }(\mathrm{cm})\end{array}$ & $\begin{array}{l}\text { Depth } \\
\text { (mbsf) }\end{array}$ & $\begin{array}{l}\mathrm{CaCO}_{3} \\
(\mathrm{wt} \%)\end{array}$ & $\underset{(\mathrm{ppm})}{\mathrm{Mg}}$ & $\underset{(\mathrm{ppm})}{\mathrm{Sr}}$ & $\underset{(\mathrm{ppm})}{\mathrm{Na}}$ & $\begin{array}{l}\delta^{13} \mathrm{C} \\
\left(\%_{00}\right)\end{array}$ & $\begin{array}{l}\delta^{18} \mathrm{O} \\
(\% 0)\end{array}$ \\
\hline \multicolumn{8}{|l|}{ 115-716B- (Cont.) } \\
\hline $27 \mathrm{H}-4,120-122$ & 250.8 & 95.8 & 2272 & 1577 & 768 & & \\
\hline $27 \mathrm{H}-4,138-141\left(^{*}\right)$ & 250.98 & 22.2 & 2207 & 71520 & 4660 & & \\
\hline $27 \mathrm{H}-5,20-22$ & 251.3 & 82.9 & 2811 & 1888 & 887 & & \\
\hline $27 \mathrm{H}-5,70-72$ & 251.8 & 95.6 & 2595 & 1599 & 675 & & \\
\hline $27 \mathrm{H}-5,120-122$ & 252.3 & 96.3 & 2645 & 1589 & 711 & & \\
\hline $27 \mathrm{H}-6,20-22$ & 252.8 & 95.5 & 2567 & 1613 & 612 & & \\
\hline $27 \mathrm{H}-6,70-72$ & 253.3 & 96.1 & 2621 & 1604 & 702 & & \\
\hline $27 \mathrm{H}-6,120-122$ & 253.8 & 95.8 & 2493 & 1725 & 596 & 0.36 & 0.22 \\
\hline $27 \mathrm{H}-7,20-22$ & 254.3 & 93.5 & 2338 & 1644 & 817 & & \\
\hline $28 \mathrm{H}-1,20-22$ & 254.9 & 90.6 & 2073 & 2128 & 605 & & \\
\hline $28 \mathrm{H}-1,70-72$ & 255.4 & 96.2 & 2043 & 1589 & 774 & & \\
\hline $28 \mathrm{H}-1,120-122$ & 255.9 & 96.4 & 1944 & 1667 & 533 & 0.38 & -0.02 \\
\hline $28 \mathrm{H}-2,20-22$ & 256.4 & 93.6 & 2081 & 1769 & 678 & & \\
\hline $28 \mathrm{H}-2,70-72$ & 256.9 & 91.7 & 2073 & 2007 & 786 & & \\
\hline $\begin{array}{c}28 \mathrm{H}-2,120-122 \\
(\mathrm{CH})\end{array}$ & 257.4 & 97.1 & 1961 & 1801 & 640 & 0.16 & 0.31 \\
\hline $28 \mathrm{H}-3,20-22$ & 257.9 & 96.4 & 2173 & 1469 & 585 & & \\
\hline $28 \mathrm{H}-3,70-72$ & 258.9 & 96.5 & 2349 & 1499 & 726 & & \\
\hline $28 \mathrm{H}-4,70-72$ & 259.9 & 94.6 & 2334 & 1681 & 622 & & \\
\hline $28 \mathrm{H}-4,120-122$ & 260.4 & 96.3 & 2380 & 1613 & 570 & & \\
\hline $28 \mathrm{H}-5,20-22$ & 260.9 & 93.2 & 2554 & 1704 & 671 & 0.38 & 0.11 \\
\hline $28 \mathrm{H}-5,70-72$ & 261.4 & 90.4 & 2739 & 1776 & 715 & & \\
\hline $28 \mathrm{H}-5,120-122$ & 261.9 & 91.7 & 2792 & 1786 & 638 & & \\
\hline $28 \mathrm{H}-6,20-22$ & 262.4 & 91.1 & 2810 & 1837 & 605 & & \\
\hline $28 \mathrm{H}-6,70-72$ & 262.9 & 95.3 & 2629 & 1746 & 638 & & \\
\hline $28 \mathrm{H}-7,20-22$ & 263.9 & 93.6 & 2643 & 1580 & 641 & & \\
\hline $28 \mathrm{H}-7,70-72$ & 264.4 & 96.4 & 2593 & 1520 & 560 & 0.39 & 0.37 \\
\hline
\end{tabular}

Note: $\mathrm{CH}=$ chalk nodule and $(*)=$ celestite nodule.

$0.48 \quad 0.28$

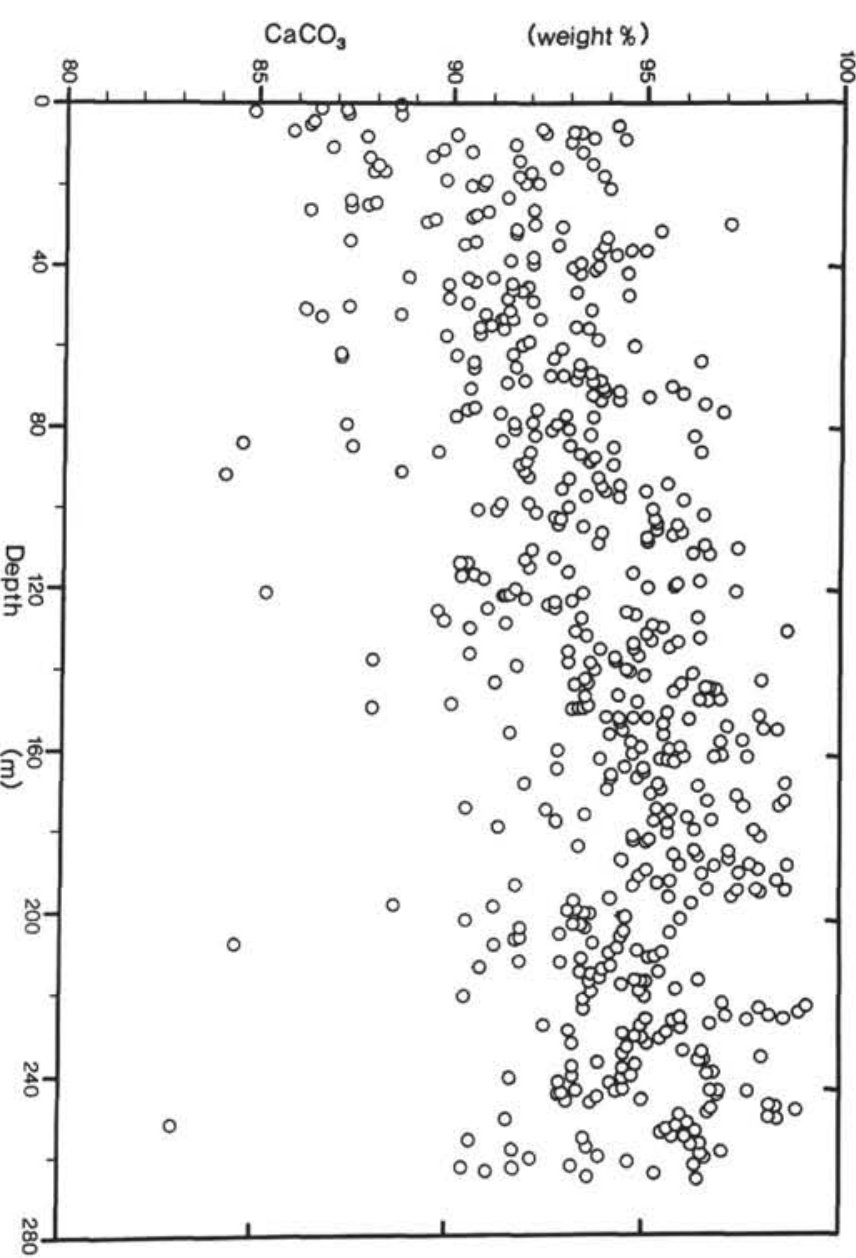

Figure 3. Calcium carbonate (wt $\%$ ) as a function of sub-bottom depth. 


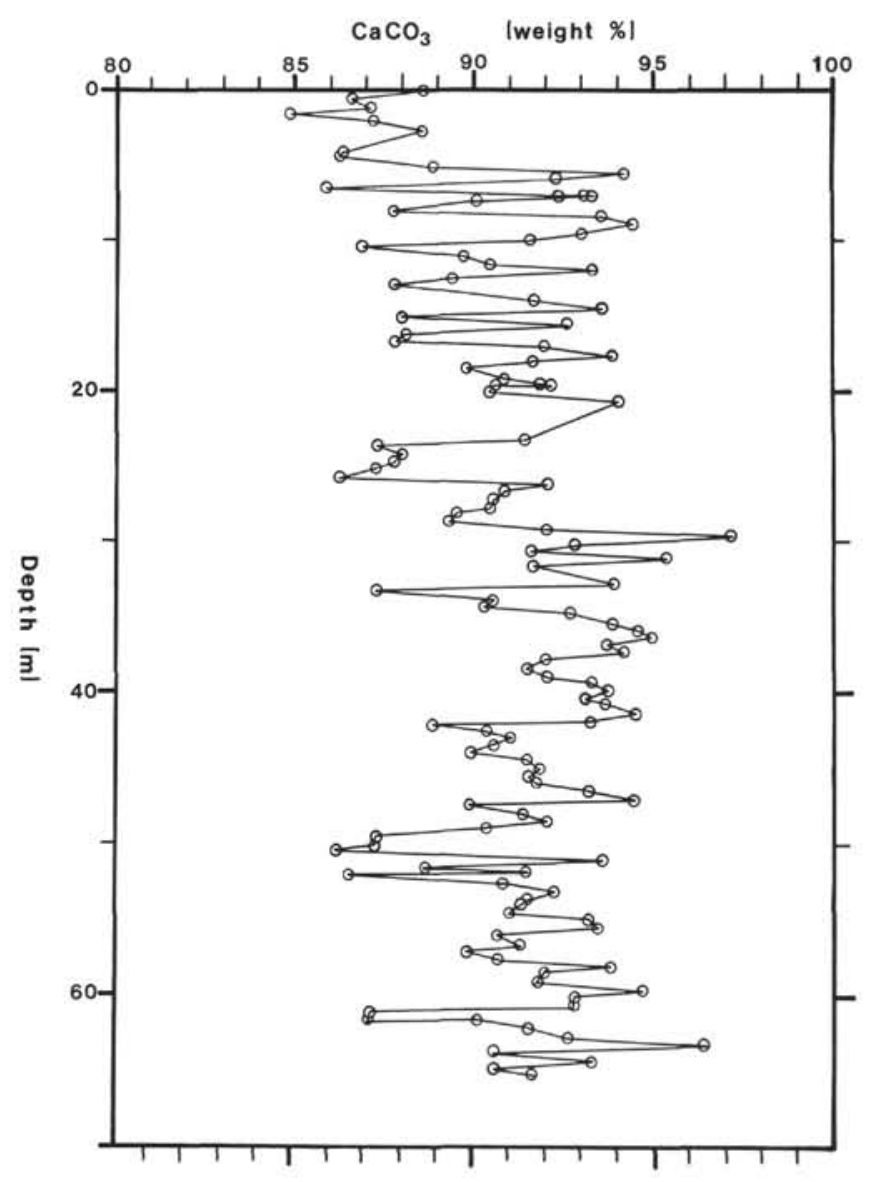

Figure 4. Calcium carbonate (wt \%) for the upper $60 \mathrm{mbsf}$.

sediments below 60 mbsf to dissolution of aragonite, HMC, and some biogenic LMC and reprecipitation of diagenetic LMC. Under these conditions, in which sulfate reduction is not excessive, dissolved strontium concentrations reach levels where pore waters are saturated or supersaturated with respect to celestite (Baker and Bloomer, 1988). At Site 716, at approximately 100 mbsf, dissolved strontium values reach maximum concentrations of about $500 \mu \mathrm{M}$ (Swart and Burns, this volume). The shallowest celestite nodule was recovered at $111.6 \mathrm{mbsf}$.

As illustrated in Figures 5 and 7, strontium and sodium concentrations behave in a similar manner, although strontium is much more variable than sodium. The sodium values gradually decrease from a maximum of $2000 \mathrm{ppm}$ at the top of the core to about $1300 \mathrm{ppm}$ at $60 \mathrm{mbsf}$. At $60 \mathrm{mbsf}$, sodium concentrations abruptly decline to values of approximately $1000 \mathrm{ppm}$ or less. An exception occurs at about 210 mbsf, where concentrations increase to $1200 \mathrm{ppm}$ and then decrease again. As with strontium, we believe the monotonic decrease in sodium is the result of the dissolution of biogenic calcium carbonate and the reprecipitation of diagenetic LMC.

Sodium concentrations in the upper $60 \mathrm{~m}$ are not as variable as strontium values in the same interval, although sodium concentrations are known to be enriched in biogenic aragonite and HMC (i.e., shallow-water algae) over LMC (foraminifers and coccoliths) (Busenberg and Plummer, 1985). We suggest that this may be related to the different chemical behavior of the two elements. Busenberg and Plummer (1985) have shown experimentally that sodium does not substitute for calcium in lattice sites in calcite but probably occupies interstitial positions in the crystal lattice. They have shown that the amount of incorpo-

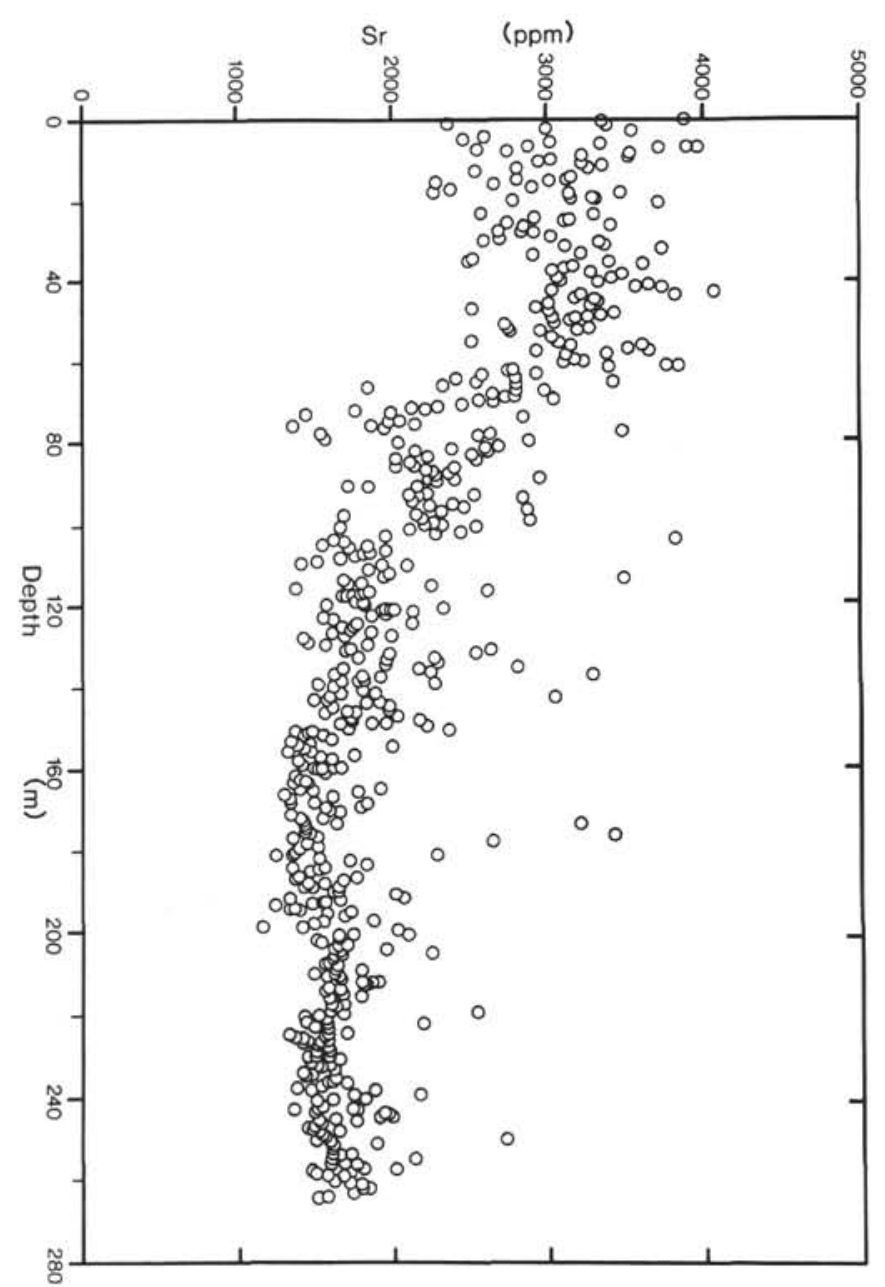

Figure 5. Strontium concentrations (ppm) in calcium carbonate as a function of sub-bottom depth.

rated sodium seems to be limited by the number of crystal defects, which, in turn, is a function of crystal growth rate and would certainly also be a function of crystal age.

Magnesium concentrations behave very differently than strontium and sodium. Magnesium values range from about 2000 to $6000 \mathrm{ppm}$ (Fig. 8). The variability in the upper $40 \mathrm{mbsf}$ is mostly attributable to the increased input of HMC from the bank-tops. The first two peaks of magnesium coincide with peaks of HMC. Below $32 \mathrm{mbsf}, \mathrm{HMC}$ is no longer present; therefore, $\mathrm{HMC}$ cannot explain the increases in magnesium. Below $40 \mathrm{mbsf}$, these cycles display a lower amplitude fluctuation, with values ranging from $\sim 2000$ to $4000 \mathrm{ppm}$. High magnesium values do not appear to be confined to the nodules but are also found in sediment between more lithified layers.

\section{Carbon and Oxygen Isotopes}

Oxygen and carbon isotope values of selected bulk samples are shown in Figures 9 and 10 and compiled in Table 1 . The $\delta^{18} \mathrm{O}$ values range from $-1.5 \%$ to $1.3 \%$, whereas $\delta^{13} \mathrm{C}$ values range from $-0.1 \%$ to $1.0 \%$. Generally, $\delta^{18} \mathrm{O}$ and $\delta^{13} \mathrm{C}$ covary downcore. Both show positive excursions at about 60 and $140 \mathrm{mbsf}$ and negative excursions at about 20,100, and $190 \mathrm{mbsf}$. The $\delta^{18} \mathrm{O}$ and $\delta^{13} \mathrm{C}$ curves also display similarity to the magnesium curve. Values of $\delta^{18} \mathrm{O}$ and $\delta^{13} \mathrm{C}$ seem to increase in intervals of increased concentrations of magnesium and in intervals of increased induration, but neither $\delta^{18} \mathrm{O}$ nor $\delta^{13} \mathrm{C}$ are significantly 


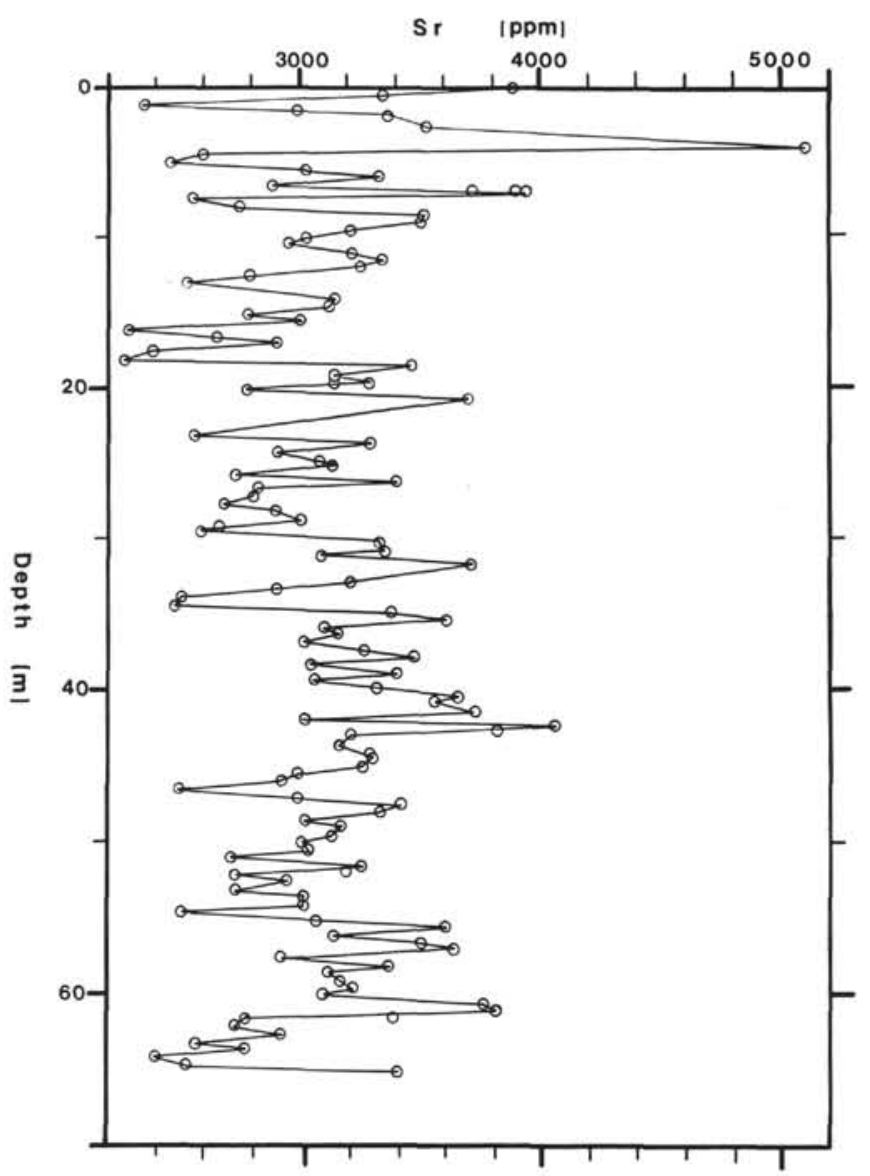

Figure 6. Strontium concentrations (ppm) in calcium carbonate for the upper $60 \mathrm{mbsf}$.

higher in discrete chalk nodules relative to neighboring sediments (Figs. 9 and 10).

\section{Scanning Electron Microscopy}

The scanning electron microscope (SEM) photomicrographs in Figure 11 illustrate the textural differences in sediments from near the seafloor in Site 716 compared with chalk horizons from the lower part of the core. Figure 11A is a nannofossil ooze from interglacial Stage 1 at 0.1 mbsf (as defined by Droxler et al., this volume). Figure 11B shows sediment from glacial Stage 2 at 0.7 mbsf. This sample is considerably coarser and consists primarily of planktonic foraminifers. Figure $11 \mathrm{C}$, from a chalk layer at $155.8 \mathrm{mbsf}$, shows rhombs of calcite cement. Figure 11D, a chalk from 257.4 mbsf near the bottom of the core, illustrates the compacted nature of the chalks from this depth.

\section{DISCUSSION}

Site 716 is unusual among all deep-sea carbonate drill sites because of its rapid lithification and shallow occurrence of chalks. In this study, we focus on the timing and nature of cementation that produced these chalks. Specifically, we will resolve whether or not the chalks formed by (1) seafloor or near-seafloor cementation, (2) burial diagenesis at an accelerated pace relative to deep-sea pelagic carbonates, or (3) a combination of both mechanisms.

\section{Seafloor Cementation}

In the Bahamas, seafloor cements are common in Pleistocene periplatform carbonate sediments (Wilbur, 1978; Mullins

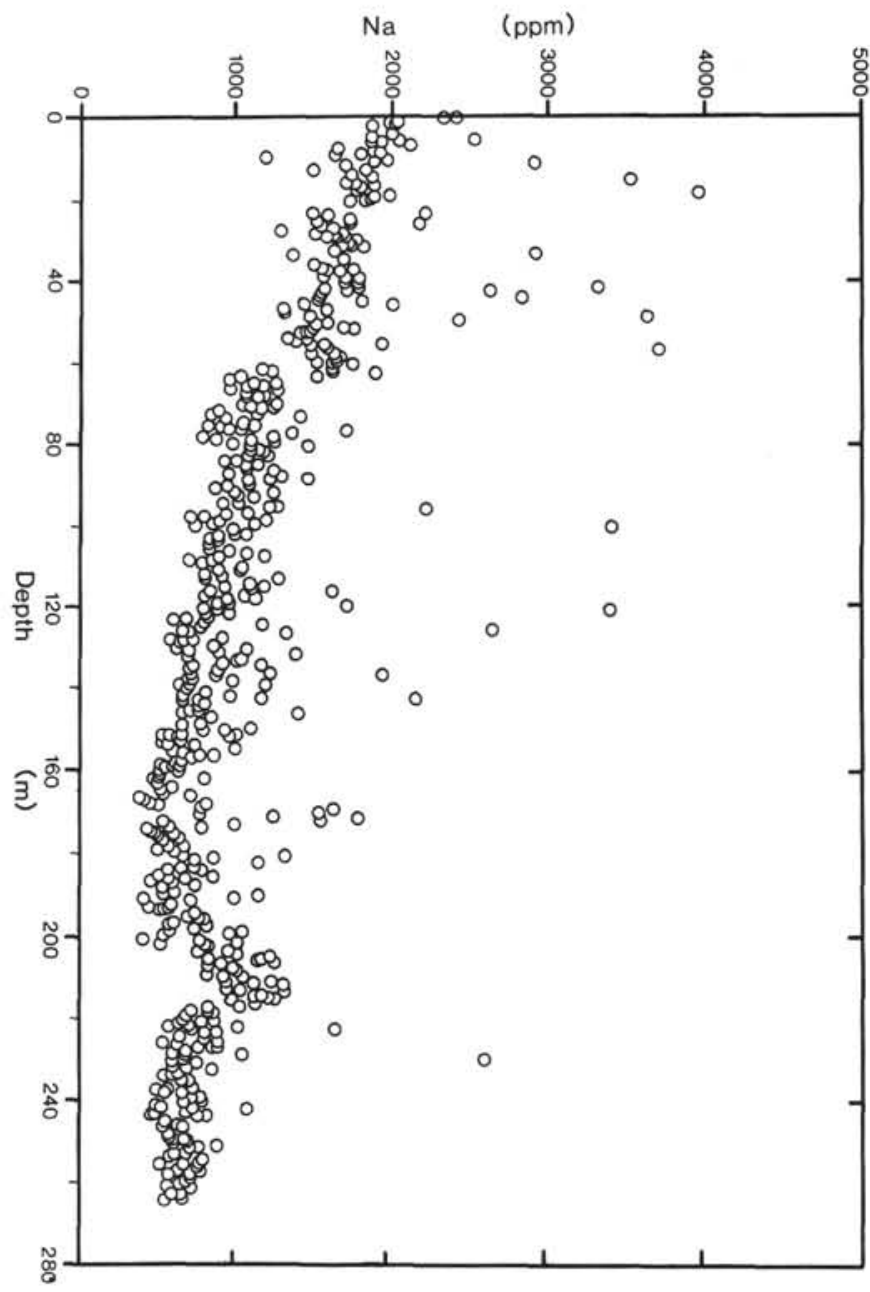

Figure 7. Sodium concentrations ( $\mathrm{ppm}$ ) in calcium carbonate as a function of sub-bottom depth.

et al., 1980; Kiefer, 1983; Slowey, 1985; Haddad, 1986). These Bahamian cements usually occur in glacial-age sediments or in glacial-to-interglacial transitions when shallow-water carbonate saturation is higher (because of decreased water depth and, therefore, increased water temperatures) and sedimentation rates are lower. Likewise, we might expect seafloor cementation in the Maldives to be more important during glacial stages than during interglacial stages. Contrary to this expectation, no chalks were found at Site 716 throughout the Pleistocene. They only occur in Pliocene and older sediments. This observation supports the hypothesis that the chalks formed during burial diagenesis.

If the chalk horizons formed at or near the sediment-water interface, then they would be expected to be isotopically heavier in oxygen and carbon than adjacent uncemented zones. Using a temperature of $10^{\circ} \mathrm{C}$ at a water depth of $500 \mathrm{~m}$ (obtained from GEOSECS Stations 447 and 448) (Weiss et al., 1983), an estimate that Indian Ocean Intermediate Water at salinity of 35.00 $\mathrm{g} / \mathrm{kg}$ has a $\delta^{18} \mathrm{O}$ of seawater $=0.0 \%$ (SMOW) and applying oxygen isotope fractionation factors between calcium carbonate and water as reported by $\mathrm{O}^{\prime}$ Neil et al. (1969), we calculate equilibrium $\delta^{18} \mathrm{O}$ of calcium carbonate cement forming on the seafloor as $0.9 \%$ (PDB). The oxygen isotopic values of bulk sediments in cemented intervals should be shifted from the assumed original isotopic composition of the sediment part way toward this predicted cement value. Figure 9 shows that, except for the three shallowest chalks, most chalks are not shifted significantly from the values of neighboring less cemented sediments. 


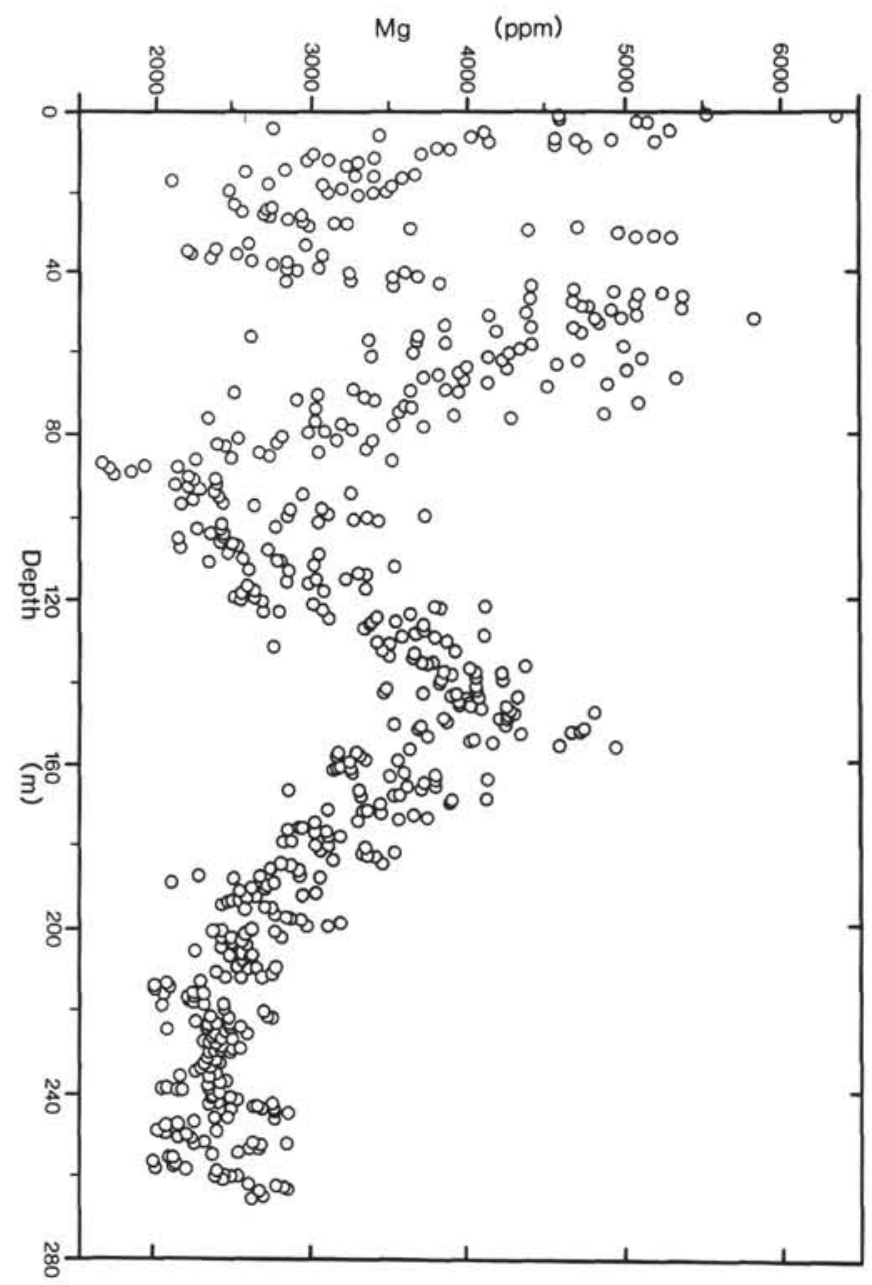

Figure 8. Magnesium concentrations (ppm) in calcium carbonate as a function of sub-bottom depth.

Using carbon isotope fractionation factors between calcium carbonate and dissolved bicarbonate reported by Emrich et al. (1970), $\delta^{13} \mathrm{C}$ values of dissolved inorganic carbon from GEOSECS Stations 447 and 448 (Kroopnick, 1985) and a temperature of $10^{\circ} \mathrm{C}$ from the same sites (Weiss et al., 1983), we calculate an equilibrium $\delta^{13} \mathrm{C}$ of $1.9 \%$ (PDB) for calcium carbonate cements forming at the seafloor. Carbon isotopic values of sediments in the cemented intervals should also be shifted part way between the assumed original isotopic composition of the sediment and predicted cement values. The $\delta^{13} \mathrm{C}$ values of chalks shown in Figure 10 are not significantly shifted toward predicted seafloor values. Thus, neither carbon nor oxygen isotopic compositions nor the depth distribution of the chalks support their origin as seafloor cements.

\section{Burial Diagenesis}

The evidence for calcium carbonate cementation during burial diagenesis is convincing. We believe that the downcore changes in mineralogy - the rapid disappearance of HMC and the more gradual decrease in aragonite-as well as the monotonic decrease of strontium and sodium are a result of the ongoing process of burial diagenesis. Droxler et al. (this volume) have shown that there is high-frequency mineralogical variation in the upper $60 \mathrm{mbsf}$ because of variations in the primary depositional record. We think that the primary record has been substantially modified by diagenesis, especially in older sediments. The down-

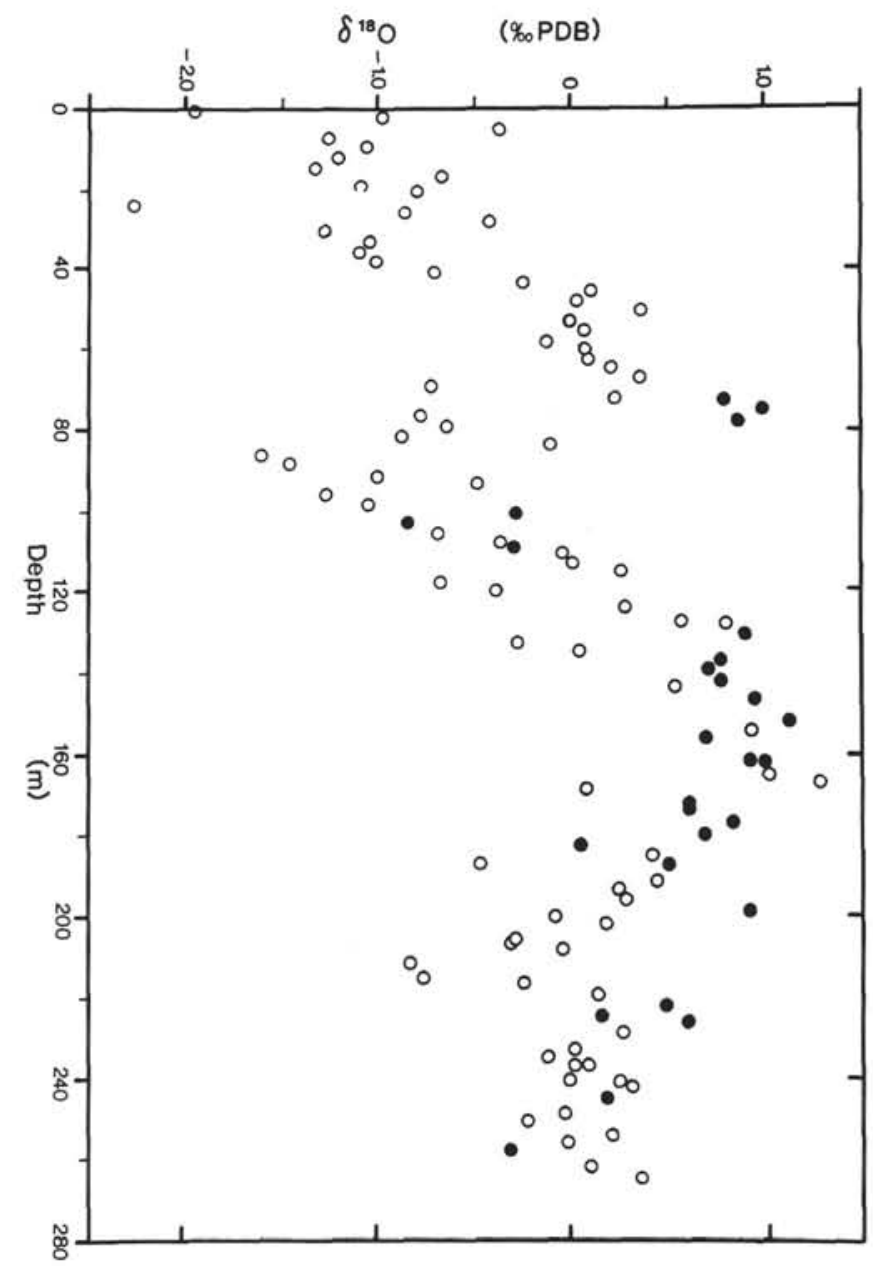

Figure $9 . \delta^{18} \mathrm{O}$ as per mil $(\% 0)$ deviations from PDB as a function of subbottom depth. Solid circles are chalks.

core decrease of strontium in the sediment and the corresponding downcore increase of dissolved strontium in the pore waters (Swart and Burns, this volume) prove that recrystallization of biogenic aragonite and calcite to inorganic calcite during burial diagenesis is occurring. Likewise, the formation of celestite nodules requires the recrystallization of biogenic calcium carbonate to increase pore-water-dissolved strontium concentrations sufficiently to allow celestite saturation (Baker and Bloomer, 1988).

Magnesium concentrations at Site 718 vary widely and do not display, in any straightforward way, trends like those observed during pelagic carbonate diagenesis (Baker et al., 1982; Renard, 1986). When LMC recrystallizes during burial diagenesis in deep-sea monomineralic calcitic oozes, normally there is a slight downcore increase of magnesium in the carbonate solids. Such changes are of much smaller magnitude and occur at far slower rates than those observed in periplatform oozes at Site 716. The HMC present in periplatform carbonate sediments converts to LMC much more rapidly, causing a decrease in the magnesium content of the sediments. Correspondingly, a small downcore increase of magnesium in the pore waters might also be expected throughout this zone. Pore-water data at Site 716 (Swart and Burns, this volume) do reveal a slight, possibly significant, increase of dissolved magnesium in the upper $40 \mathrm{mbsf}$.

Below the zone containing HMC, there are two intervals of highly elevated magnesium values in the bulk sediment. The magnesium content of these intervals has two likely origins: either these zones are composed of a small amount (about 10\%) 


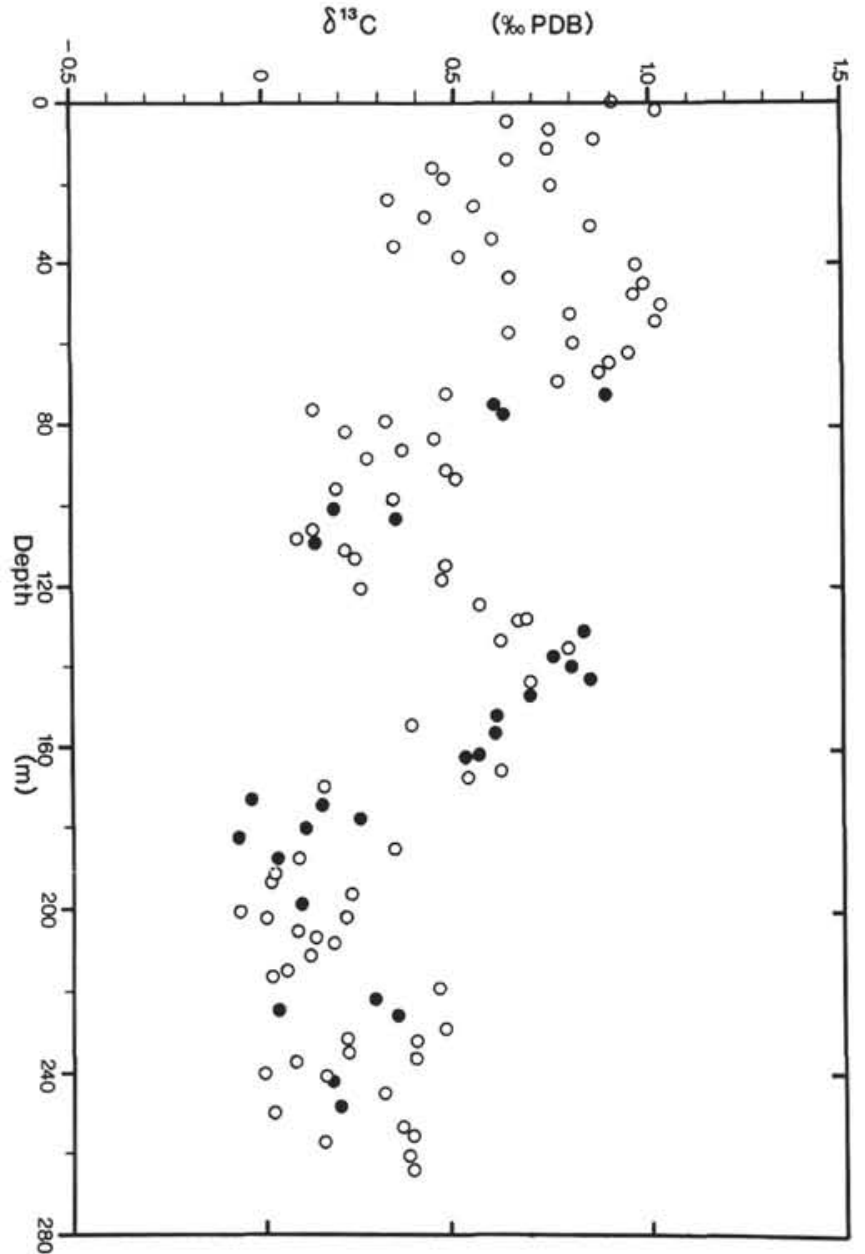

Figure 10. $\delta^{13} \mathrm{C}$ as per mil (\%) deviations from PDB as a function of sub-bottom depth. Solid circles are chalks.

of HMC cement or they are composed of a large amount of only mildly enriched LMC cement. Because XRD results reveal no HMC below $32 \mathrm{mbsf}$, the second hypothesis is favored. If we assume that these intervals contain about $30 \%$ cement, then these cements are composed of about 3-4 mol\% magnesium. Figures 2 and 8 show that magnesium and aragonite generally covary downcore. Consequently, these two magnesium peaks could represent a vestige of the former presence of HMC, coincident with the aragonite highs in the original sediment. This implies that the originally high percentage of HMC and its subsequent conversion to LMC in these layers of sediment resulted in accelerated rates of recrystallization. The resultant recrystallized LMC retained much of the original magnesium and, therefore, is mildly enriched relative to less-cemented intervals. Thus, magnesium contents in older periplatform carbonate sediments may also be expected to record past elevated inputs of HMC, and possibly aragonite, as well as past high stands of sea level.

As previously noted, $\delta^{13} \mathrm{C}$ and magnesium covary below 40 mbsf. Therefore, the coherent variations in $\delta^{13} \mathrm{C}$ may also represent variations in sedimentary input from the banks. Bank-derived biogenic aragonite, such as green algae, is enriched in ${ }^{13} \mathrm{C}$, usually ranging from $2.0 \%$ to $5.0 \%$ (PDB) (Milliman, 1974). Figures 2 and 10 show that positive excursions of $\delta^{13} \mathrm{C}$ are associated with increases in aragonite. This relationship is especially apparent between 120 and 160 mbsf. Because $\delta^{13} \mathrm{C}$ covaries with the aragonite content of the present sediments, $\delta^{13} \mathrm{C}$ variations may also reflect an isotopic signature inherited from formerly present aragonite and HMC recrystallized to LMC.

Differences between $\delta^{18} \mathrm{O}$ of pelagic and shallow water components are generally much less however, (Milliman, 1974) and cannot explain the variations in $\delta^{18} \mathrm{O}$ at this site. It is also well known that the original $\delta^{13} \mathrm{C}$ record is better preserved than the original $\delta^{18} \mathrm{O}$ record, because most of the carbon in sediments is found in calcium carbonate whereas most of the oxygen is in water. If we assume a geothermal gradient of $25^{\circ} \mathrm{C} / \mathrm{km}$ for Site 716 , then recrystallization during burial diagenesis must have occurred between $\sim 10^{\circ}$ and $17^{\circ} \mathrm{C}$. Therefore, carbonate sediments equilibrated with colder waters than waters in which they formed. Consequently, an increase of $\delta^{18} \mathrm{O}$ is expected to occur during isotopic equilibration, and intervals of greater recrystallization should be characterized by positive shifts of $\delta^{18} \mathrm{O}$. Therefore, at increased burial depths, $\delta^{18} \mathrm{O}$ values increasingly reflect the process of recrystallization and increasingly covary with the magnesium and $\delta^{13} \mathrm{C}$ curves.

\section{CONCLUSIONS}

Stabilization of metastable carbonates at Site 716 is rapid. HMC disappears and is reprecipitated as LMC within $1.1 \mathrm{Ma}$, whereas most aragonite has disappeared and is converted to LMC by $2.5 \mathrm{Ma}$ and is completely gone by $6 \mathrm{Ma}$. The youngest celestites (in equilibrium with modern-day pore waters) occur in sediments of approximately $3.4 \mathrm{Ma}$, coincident with the subbottom depth of the maximum rate of decrease of solid strontium concentrations. Strontium concentrations in carbonate solids decrease with depth in response to burial diagenesis. Sodium concentrations in carbonate solids rapidly decrease with depth and are also controlled by burial diagenesis.

Magnesium concentrations vary widely. In sediments younger than $1.1 \mathrm{Ma}$, magnesium concentration variations are controlled by the amount of HMC present. Intervals of increased magnesium values in sediments older than $1.1 \mathrm{Ma}$ are a result of episodes of cementation during burial diagenesis. These intervals are controlled by the former higher abundance of metastable platform-derived aragonite and HMC. Carbon isotopes record these changes in sedimentary input from shallow-water banks. Primary carbon isotopic compositions are retained despite extensive burial diagenesis. Oxygen isotopes also record a primary paleoceanographic signal in sediments younger than approximately $2 \mathrm{Ma}$, but the $\delta^{18} \mathrm{O}$ values of sediments older than $2 \mathrm{Ma}$ (below $60 \mathrm{mbsf}$ ) increasingly reflect equilibration of carbonates with cold pore waters during diagenetic dissolution and reprecipitation. These conclusions have important implications for the diagenesis of ancient shallow-water limestones:

1. Biogenic HMC recrystallizes faster than biogenic aragonite, which recrystallizes faster than biogenic LMC. Rates of recrystallization at this site are $1 \mathrm{Ma}$ for $\mathrm{HMC}$ and 2.5-6 Ma for aragonite.

2. Magnesium is, at least partially, retained during the recrystallization of HMC in marine pore fluids. Hence, high magnesium values found in ancient limestones may be caused by an original high content of HMC. In contrast, strontium does not seem to be retained much during the dissolution of aragonite and reprecipitation of LMC.

3. In periplatform carbonate sediments or limestones, carbon isotopic highs may be the result of increased inputs of shallow-water-derived aragonitic components. These values are retained during marine burial diagenesis. Oxygen isotopic changes in the sediments record the presence of sub-bottom cements. It is expected that oxygen isotopic values will be overprinted by later cementation. 


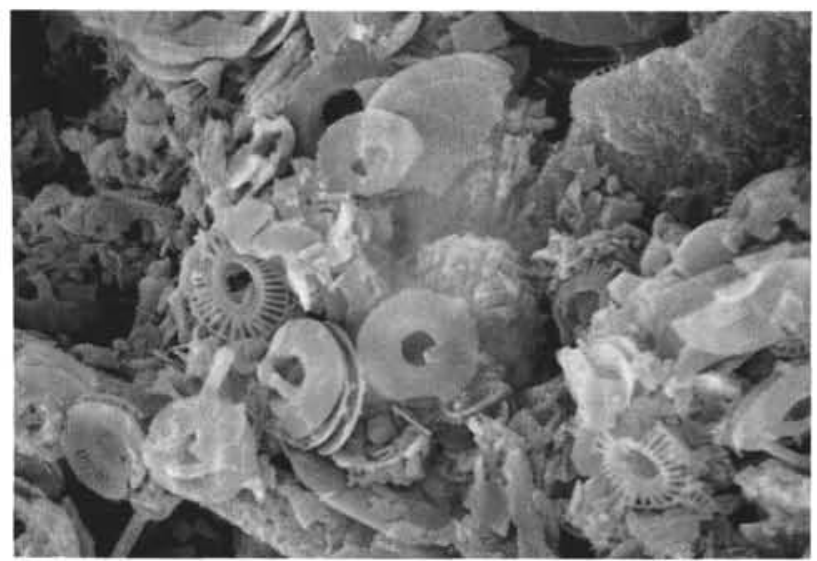

A

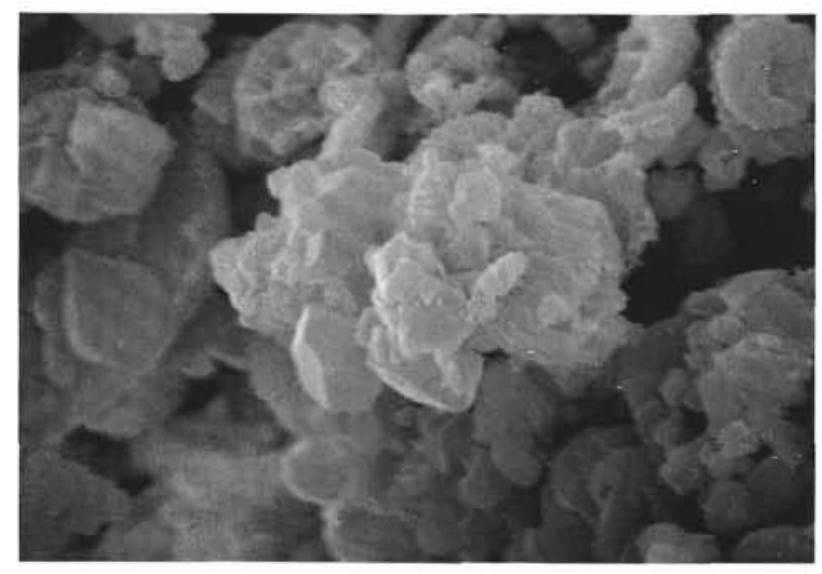

C

$10 \mu \mathrm{m}$

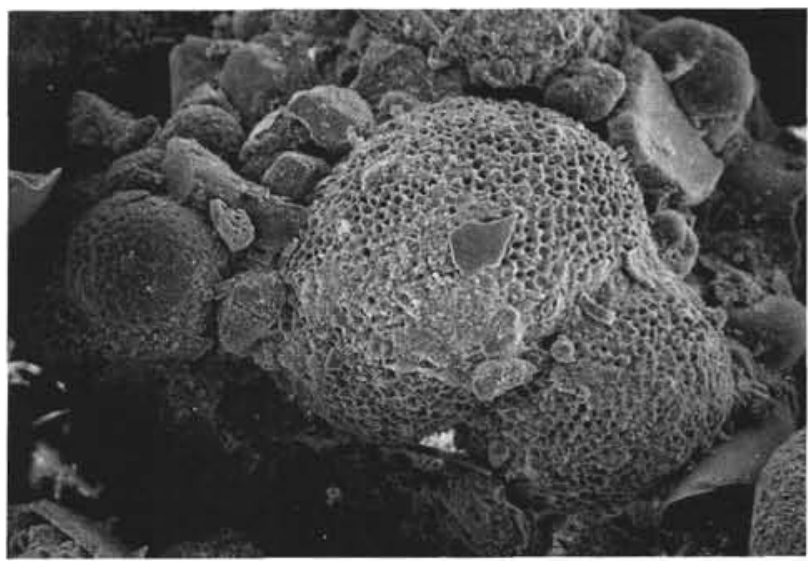

B

$\overline{100 \mu \mathrm{m}}$

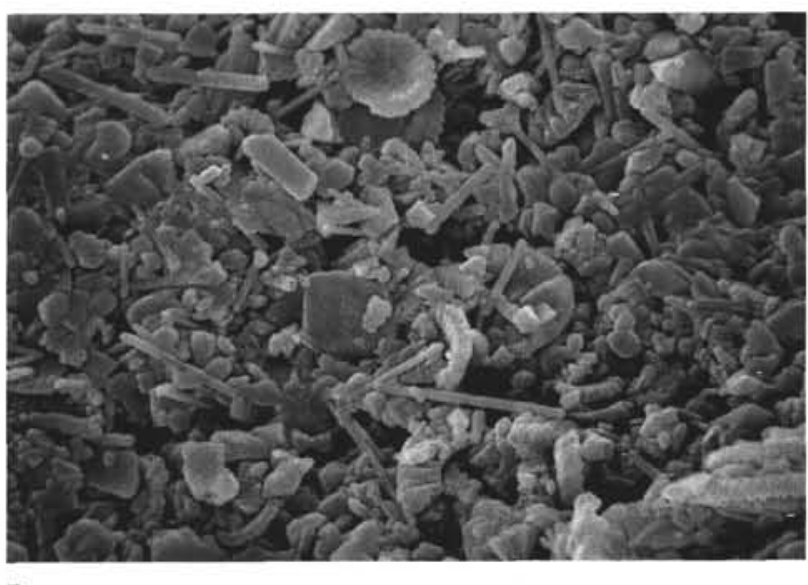

D

$10 \mu \mathrm{m}$

Figure 11. SEM photomicrographs. A. Nannofossil ooze ( $0.1 \mathrm{mbsf})$. B. Planktonic foraminifers $(0.7 \mathrm{mbsf})$. C. Chalk horizon showing calcite cement (155.8 mbsf). D. Chalk horizon (257.4 mbsf).

\section{ACKNOWLEDGMENTS}

We thank the crew and the rest of the shipboard technical and scientific parties, especially the co-chief scientists, J. Backman and R. A. Duncan. The help of all these people was essential for the present study. We thank A. Droxler and G. Haddad for their help in sampling and providing preliminary data and reviews. We also thank $\mathrm{M}$. Malone for assistance in sampling. The comments of two anonymous reviewers were very helpful. We acknowledge the support by USSAC and National Science Foundation Grant No. OCE-87-18382 to P. A. Baker.

\section{REFERENCES}

Backman, J., Duncan, R. A., et al., 1988. Proc. ODP, Init. Repts., 115: College Station, TX (Ocean Drilling Program).

Baker, P. A., 1981. The diagenesis of marine carbonate sediments: experimental and natural observations [Ph.D. dissert.]. Univ. California, San Diego.

Baker, P. A., and Bloomer, S. H., 1988. The origin of celestite in deepsea carbonate sediments. Geochim. Cosmochim. Acta, 52:335-340.

Baker, P. A., Gieskes, J. M., and Elderfield, H., 1982. Diagenesis of carbonates in deep-sea sediments-evidence from $\mathrm{Sr} / \mathrm{Ca}$ ratios and interstitial dissolved $\mathrm{Sr}^{2+}$ data. J. Sediment. Petrol., 52:71-82.
Boardman, M. R., and Neumann, A. C., 1984. Sources of periplatform carbonates: Northwest Providence Channel, Bahamas. J. Sediment. Petrol., 54:110-1123.

Boardman, M. R., Neumann, A. C., Baker, P. A., Dulin, L. A., Kenter, R. J., Hunter, G. E., and Kiefer, K. B., 1986. Bank-top response to Quaternary fluctuations in sea level recorded in periplatform sediments. Geology, 14:28-31.

Burns, S. J., and Neumann, A. C., 1987. Pelagic sedimentation on an inactive gullied slope, Northwest Providence Channel, Bahamas. Mar. Geol., 77:277-286.

Busenberg, E., and Plummer, L. N., 1985. Kinetic and thermodynamic factors controlling the distribution of $\mathrm{SO}_{4}^{2-}$ and $\mathrm{Na}^{+}$in calcites and selected aragonites. Geochim. Cosmochim. Acta, 49:713-725.

Dix, G. R., and Mullins, H. T., 1988a. A regional perspective of shallow-burial diagenesis of deep-water periplatform carbonates from the Northern Bahamas. In Austin, J. A., Jr., Schlager, W., et al., Proc. ODP, Sci. Results, 101: College Station, TX (Ocean Drilling Program), 279-302.

1988b. Rapid burial diagenesis of deep-water carbonates: Exuma Sound, Bahamas. Geology, 16:680-683.

Droxler, A. W., Schlager, W., and Wallon, C. C., 1983. Quaternary aragonite cycles and oxygen-isotope record in Bahamian carbonate ooze. Geology, 11:235-239.

Droxler, A. W., Bruce, C. H., Sager, W. W., and Watkins, D. H., 1988. Pliocene-Pleistocene variations in aragonite content and planktonic 
oxygen-isotope record in Bahamian periplatform ooze, Hole 633A. In Austin, J. A., Jr., Schlager, W., et al., Proc. ODP, Sci. Results, 101: College Station, TX (Ocean Drilling Program), 221-244.

Emrich, K., Ehhalt, D., and Vogel, J., 1970. Carbon isotope fractionation during the precipitation of calcium carbonate. Earth Planet. Sci. Lett., 8:363-371.

Gealy, E. L., Winterer, E. L., and Moberly, R., Jr., 1971. Methods, conventions, and general observations. In Winterer, E. L., Riedel, W. R., et al., Init. Repts. DSDP, 7, Pt. 1: Washington (U.S. Govt. Printing Office), 9-26.

Haddad, G. A., 1986. A study of carbonate dissolution, stable isotope chemistry and minor element composition of pteropods and forams deposited in the Northwest Providence Channel, Bahamas during the past 500,000 years [M.S. thesis]. Duke Univ., Durham, NC.

Kiefer, K., 1983. Quaternary climatic cycles recorded in the isotopic record of periplatform pelagic deposition [M.S. thesis]. Duke Univ., Durham, NC.

Kier, J. S., and Pilkey, O. H., 1971. The influence of sea-level changes on sediment carbonate mineralogy, Tongue of the Ocean, Bahamas. Mar. Geol., 11:189-200.

Kroopnick, P., 1985. The distribution of ${ }^{13} \mathrm{C}$ of $\Sigma^{2} \mathrm{CO}_{2}$ in the world oceans. Deep-Sea Res., 32:57-84.

Milliman, J. D., 1974. Marine Carbonates (2nd ed.): Berlin-HeidelbergNew York (Springer-Verlag).

Mullins, H. T., Neumann, A. C., Wilbur, R. J., and Boardman, M. R., 1980. Nodular carbonate sediment on Bahamian slopes: possible precursors to nodular limestones. J. Sediment. Petrol., 50:117-131.

Mullins, H. T., Wise, S. W., Jr., Gardulski, A. F., Hinchey, E. J., Masters, P. M., and Siegel, D. I., 1985a. Shallow subsurface diagenesis of Pleistocene periplatform ooze: northern Bahamas. Sedimentology, 32:473-494.
Mullins, H. T., Wise, S. W., Jr., Land, L. S., Siegel, D. I., Masters, P. M., Hinchey, E. J., and Price, K. R., 1985b. Authigenic dolomite in Bahamian periplatform slope sediment. Geology, 13:292-295.

O'Neil, J. R., Clayton, R. E., and Mayeda, T. K., 1969. Oxygen isotope fractionation in divalent metal carbonates. J. Chem. Physics, 51: $5547-5558$.

Renard, M., 1986. Pelagic carbonate chemostratigraphy $\left(\mathrm{Sr}, \mathrm{Mg},{ }^{18} \mathrm{O}\right.$, ${ }^{13}$ C). Mar. Micropaleontol., 10:117-164.

Schlager, W., and James, N. P., 1978. Low-magnesian calcite limestones forming at the deep-sea floor Tongue of the Ocean, Bahamas. Sedimentology, 25:675-702.

Slowey, N. C., 1985. Fine-scale acoustic stratigraphy of Northwest Providence Channel, Bahamas [M.S. thesis]. Univ. North Carolina, Chapel Hill, NC.

Swart, P. K., and Guzikowski, M., 1988. Interstitial-water chemistry and diagenesis of periplatform sediments from the Bahamas, ODP Leg 101. In Austin, J. A., Jr., Schlager, W., et al., Proc. ODP, Sci. Results, 101: College Station, TX (Ocean Drilling Program), 363380.

Weiss, R., Broecker, W., Craig, H., and Spencer, D., 1983. GEOSECS Atlas. Indian Ocean Expedition: Hydrographic Data (Vol. 5): Washington (U.S. Govt. Printing Office).

Wilbur, R. J., 1976. Petrology of submarine-lithified hardgrounds and lithoherms from their deep flank environment of the little Bahama Bank (Northeast Straits of Florida) [M.S. thesis]. Duke Univ., Durham, NC.

Date of initial receipt: 12 June 1989

Date of acceptance: 5 January 1990

Ms 115B-184 